\title{
Weak containment in the space of actions of a free group
}

\author{
Alexander S. Kechris
}

(A) We consider measure preserving actions of an infinite, countable (discrete) group $\Gamma$ on non-atomic standard measure spaces $(X, \mu)$, i.e., standard Borel spaces equipped with a non-atomic probability Borel measure. (All such measure spaces are isomorphic to $([0,1], \lambda)$, where $\lambda$ is Lebesgue measure.) We denote by $A(\Gamma, X, \mu)$ the space of such actions. If $a \in A(\Gamma, X, \mu)$ and $\gamma \in \Gamma$, we denote by $\gamma^{a}(x)=a(\gamma, x)$, the corresponding automorphism of the space $(X, \mu)$. The group $\operatorname{Aut}(X, \mu)$ admits a canonical Polish topology, called the weak topology, which is the topology generated by the maps $T \mapsto T(A)$ ( $A$ a Borel subset of $X)$ from $\operatorname{Aut}(X, \mu)$ to the measure algebra $\operatorname{MALG}(X, \mu)$ of $(X, \mu)$, equipped with the metric $d_{\mu}(A, B)=\mu(A \Delta B)$ and the corresponding topology. Since $A(\Gamma, X, \mu)$ can be viewed as a subspace of the produced space $\operatorname{Aut}(X, \mu)^{\Gamma}$, it inherits the product of the weak topology which we also call the weak topology on $A(\Gamma, X, \mu)$. Note that $\operatorname{Aut}(X, \mu)$ acts continuously via conjugation on $A(\Gamma, X, \mu)$ : Given $S \in \operatorname{Aut}(X, \mu)$ and $a \in A(\Gamma, X, \mu)$, we let $S \cdot a=S a S^{-1}$ be the action of $\Gamma$ for which $\gamma^{S a S^{-1}}=$ $S \gamma^{a} S^{-1}, \forall \gamma \in \Gamma$. Then $a, b \in A(\Gamma, X, \mu)$ are conjugate iff they are isomorphic, in symbols $a \cong b$.

Motivated by the concept of weak containment of unitary representations, we can consider an analogous concept of weak containment of actions (see Kechris [Ke09], Section 10, (C)). We say, for $a \in A(\Gamma, X, \mu), b \in A(\Gamma, Y, \nu)$, that $a$ is weakly contained in $b$, in symbols

$$
a \prec b,
$$

if for any Borel sets $A_{1}, \ldots, A_{n} \subseteq X, \gamma_{1} \ldots \gamma_{m} \in \Gamma$ and $\epsilon>0$, there are Borel 
sets $B_{1}, \ldots, B_{n} \subseteq X$ such that

$$
\left|\mu\left(\gamma_{i}^{a}\left(A_{j}\right) \cap A_{k}\right)-\nu\left(\gamma_{i}^{b}\left(B_{j}\right) \cap B_{k}\right)\right|<\epsilon,
$$

$\forall i \leq m, \forall j, k \leq n$.

Alternatively one can see that the following are equivalent for any $a \in$ $A(\Gamma, X, \mu), b \in A(\Gamma, Y, \nu)$ :

(i) $a \prec b$,

(ii) $a$ is in the weak closure of $\{c \in A(\Gamma, X, \mu): c \cong b\}$.

If $(Y, \nu)=(X, \mu)$, these are also clearly equivalent to:

(iii) $a$ is in the weak closure of the conjugacy class of $b$.

(See Kechris [Ke09], Section 10 (C).)

We say that $a, b$ are weakly equivalent if $a \prec b$ and $b \prec a$. It is easy to see that $\prec$ is a partial (pre-)order on $A(\Gamma, X, \mu)$. It was shown independently by Hjorth (unpublished) and Glasner-Thouvenot-Weiss [GTW] that $\prec$ has a largest element (unique up to weak equivalence), which we denote by $a_{\infty}$. This means that $a_{\infty}$ has dense conjugacy class in $A(\Gamma, X, \mu)$. The action $a_{\infty}$ is obtained by taking the (diagonal) product of a countable dense set $\left\{a_{n}\right\}$ of actions in $A(\Gamma, X, \mu)$, but this hardly gives a concrete representation of $a_{\infty}$. It is thus of interest to "compute" explicitly such maximum actions for various groups $\Gamma$. We do that below for the free groups $\mathbb{F}_{n}$. An additional motivation for this goal is the connection with the theory of costs, which will be described below.

If $\Gamma$ is an infinite, residually finite group, then $\Gamma$ is a dense subgroup of its profinite completion $\hat{\Gamma}$ which is defined as the inverse limit of the groups $\Gamma / N, N \triangleleft \Gamma,[\Gamma: N]<\infty$. Clearly $\hat{\Gamma}$ is a compact Polish group and we let $\hat{\eta}_{\Gamma}$ be its (normalized) Haar measure, which is clearly non-atomic. The (left-) translation action of $\Gamma$ on $\hat{\Gamma}$ is a measure preserving action of $\Gamma$ on $\left(\hat{\Gamma}, \hat{\eta}_{\Gamma}\right)$, which we denote by $p_{\Gamma}$. We now have:

Theorem 1 Let $1 \leq n \leq \infty$ and let $\mathbb{F}_{n}$ be the free group with $n$ generators. Then the action $p_{\mathbb{F}_{n}}$ is maximum in the order $\prec$ of weak containment of measure preserving actions of $\mathbb{F}_{n}$.

We now discuss an application to the theory of costs by showing how Theorem 1 together with the result in Abert-Nikolov [AN] gives a new method 
for showing that the cost of any free, measure preserving action of $\mathbb{F}_{n}(1 \leq$ $n<\infty$ ) is equal to $n$, a result originally proved by Gaboriau [G]. (Recall that an action $a \in A(\Gamma, X, \mu)$ is free if $\gamma^{a}(x) \neq x, \forall \gamma \neq 1, \mu$-a.e. $(x)$.)

Denote by $\operatorname{FR}(\Gamma, X, \mu)$ the subspace of $A(\Gamma, X, \mu)$ consisting of the free actions. For any $a \in A(\Gamma, X, \mu)$, we write

$$
x E_{a} y \Leftrightarrow \exists \gamma\left(\gamma^{a}(x)=y\right),
$$

for the equivalence relation induced by $a$. We denote by $C_{\mu}\left(E_{a}\right) \equiv C\left(E_{a}\right)$ the cost of the equivalence relation $E_{a}$, see Gaboriau [G]. Finally, we put

$$
C_{\mu}(a) \equiv C(a)=C\left(E_{a}\right) .
$$

Then $C: A(\Gamma, X, \mu) \rightarrow[0, \infty]$. We now have:

Theorem 2 (Kechris [Ke09], 10.13) If $\Gamma$ is infinite and finitely generated, then $C \mid \operatorname{FR}(\Gamma, X, \mu)$ is upper semicontinuous.

Corollary 3 For such $\Gamma$, if $a, b \in \operatorname{FR}(\Gamma, X, \mu)$, then

$$
a \prec b \Rightarrow C(b) \leq C(a) .
$$

The cost of the group $\Gamma$ is defined by

$$
C(\Gamma)=\inf \{C(a): a \in \operatorname{FR}(\Gamma, X, \mu)\} .
$$

It thus follows that if $a_{\infty}$ is maximum in the order $\prec$, then

$$
C(\Gamma)=C\left(a_{\infty}\right),
$$

which gives an additional reason for "computing" explicitly $a_{\infty}$. For the case of the free groups, we now have:

Corollary 4 For $1 \leq n<\infty, C\left(\mathbb{F}_{n}\right)=C\left(p_{\mathbb{F}_{n}}\right)$.

Now Abert-Nikolov [AN] had already found an explicit calculation of the cost of $p_{\Gamma}$, which is as follows.

Theorem 5 (Abert-Nikolov [AN]) Let $\Gamma$ be an infinite, finitely generated, residually finite group and let $\mathrm{RG}(\Gamma)$ be its (absolute) rank gradient:

$$
\operatorname{RG}(\Gamma)=\inf _{[\Gamma: H]<\infty} \frac{d(H)-1}{[\Gamma: H]},
$$

where $d(H)$, the rank of $H$, is the smallest number of generators of $H$. Then

$$
C\left(p_{\Gamma}\right)=\operatorname{RG}(\Gamma)+1 .
$$


Now in the case $\Gamma=\mathbb{F}_{n}(1 \leq n<\infty)$, it is a standard fact in group theory that for any $H \leq \Gamma$ with $[\Gamma: H]<\infty$, we have

$$
\frac{d(H)-1}{[\Gamma: H]}=n-1
$$

SO

$$
C\left(\mathbb{F}_{n}\right)=C\left(p_{\mathbb{F}_{n}}\right)=n .
$$

Also, since clearly for every $a \in \mathrm{FR}\left(\mathbb{F}_{n}, X, \mu\right)$, we have $C(a) \leq n$, it follows that $C(a)=n$, for every $a \in \mathrm{FR}\left(\mathbb{F}_{n}, X, \mu\right)$, i.e., $\mathbb{F}_{n}$ has fixed price.

(B) It is now of some interest to investigate for which residually finite groups $\Gamma$ the analog of Theorem 1 goes through, i.e., $p_{\Gamma}$ is maximum in the order of weak containment. It is also of some interest to investigate the (a priori) weaker condition that the (diagonal) product action $i_{\Gamma} \times p_{\Gamma}$, where $i_{\Gamma}$ is the trivial action of $\Gamma$ (on a non-atomic standard measure space) is maximum in $\prec$. Note that $p_{\Gamma} \prec i_{\Gamma} \times p_{\Gamma}$ and $i_{\Gamma} \times p_{\Gamma}$ is the direct sum of continuum many copies of $p_{\Gamma}$. Moreover $C\left(p_{\Gamma}\right)=C\left(i_{\Gamma} \times p_{\Gamma}\right)$ (see KechrisMiller $[\mathrm{KM}], 18.14$ ). We introduce the following terminology (whose choice will be explained below).

An infinite, residually finite group $\Gamma$ has the property $\mathrm{MD}$ if $i_{\Gamma} \times p_{\Gamma}$ is maximum in the order $\prec$ of weak containment and has the property EMD if $p_{\Gamma}$ is maximum.

It turns out that each one of these properties is equivalent to an appropriate density condition in the space $A(\Gamma, X, \mu)$.

An action $a \in A(\Gamma, X, \mu)$ is called finitely modular or profinite if there is a decreasing sequence of finite Borel partitions $\{X\}=\mathcal{P}_{0} \geq \mathcal{P}_{1} \geq \ldots$ such that each $\mathcal{P}_{n}$ is $\Gamma$-invariant and $\left\{\mathcal{P}_{n}\right\}$ separates points. Up to isomorphism, these can be also equivalently described as the actions of the following form: Given an infinite, finite splitting, rooted tree $T$ with no finite branches, and an action of $\Gamma$ by automorphisms on $T$, let $\partial T$ be the boundary of $T$ and consider the induced action of $\Gamma$ on $\partial T$ and a (non-atomic) invariant measure $\mu$ on $\partial T$ for this action. Then the finitely modular actions, up to isomorphism, are exactly the actions of $\Gamma$ on $(\partial T, \mu)$ as above. The finitely modular actions which are ergodic correspond exactly to the actions of $\Gamma$ on trees which are level transitive, i.e., act transitively on each level of the tree (see $§ 1$ below for details).

Finally, let us call $a \in A(\Gamma, X, \mu)$ finite iff it factors through an action of a finite group, i.e., there is a finite group $\Delta$, an action $b \in A(\Delta, X, \mu)$, and a surjective homomorphism $\pi: \Gamma \rightarrow \Delta$, such that $\gamma^{a}=\pi(\gamma)^{b}, \forall \gamma \in \Gamma$. 
We now have

Proposition 6 The following are equivalent for each infinite, residually finite group $\Gamma$ :

i) $\Gamma$ satisfies EMD,

ii) The ergodic, finitely modular actions are dense in $A(\Gamma, X, \mu)$.

Also the following are equivalent:

a) $\Gamma$ satisfies $\mathrm{MD}$,

b) The finitely modular actions are dense in $A(\Gamma, X, \mu)$,

c) The finite actions are dense in $A(\Gamma, X, \mu)$.

Clearly

$$
\mathrm{EMD} \Rightarrow \mathrm{MD}
$$

and any group that satisfies EMD cannot have property $(\tau)$ (which states that the trivial representation $1_{\Gamma}$ is weakly contained in the Koopman representation associated to $p_{\Gamma}$, restricted to the orthogonal of the constants). Moreover, from a recent result of Abert-Elek [AE], it follows that when $\Gamma$ does not have property $(\tau)$, then $\Gamma$ satisfies EMD iff it satisfies MD.

It is not known if EMD and MD are equivalent but by the above remarks this question is equivalent to the problem of whether the property MD and $(\tau)$ are incompatible. It is also unknown whether MD and property $(\mathrm{T})$ are incompatible.

The property MD is an ergodic theoretic analog of the property FD discussed in Lubotzky-Shalom [LS] (see also Lubotzky-Zuk [LZ]). This asserts that the finite unitary representations of $\Gamma$ on an infinite-dimensional separable Hilbert space $\mathcal{H}$ are dense in the $\operatorname{space} \operatorname{Rep}(\Gamma, \mathcal{H})$ of unitary representations of $\Gamma$ in $\mathcal{H}$. Here $\operatorname{Rep}(\Gamma, \mathcal{H}) \subseteq U(\mathcal{H})^{\Gamma}$, where $U(\mathcal{H})$ is the unitary group of $\mathcal{H}$, is equipped with the product topology, with $U(\mathcal{H})$ having the weak topology. (A finite representation is again one that factors through a representation of a finite group.) One can see that

$$
\mathrm{MD} \Rightarrow \mathrm{FD}
$$

but the converse is not known. It is also not known if FD is incompatible with property $(\mathrm{T})$ or property $(\tau)$. 
The extent of the class of infinite, residually finite groups that satisfy EMD or MD is rather unclear. We have seen that the free groups $\mathbb{F}_{n}$ satisfy EMD and so do all amenable groups. Moreover the property MD is stable under going to subgroups (but this is not clear for EMD) and both EMD, MD are stable under going to supergroups of finite index. Thus, in particular, $\mathrm{SL}_{2}(\mathbb{Z})$ and $A * B$, for $A, B$ finite non-trivial groups, have property EMD. On the other hand Lubotzky-Shalom [LSh] and Lubotzky-Zuk [LZ] discuss various examples, including $\mathrm{SL}_{n}(\mathbb{Z})$, for $n \geq 3$, that fail to have property $\mathrm{FD}$ and thus also fail to have property MD.

(C) It turns out that there is also another description of the maximum, under weak containment, action of a free group.

Theorem 7 Let $\Gamma=\mathbb{F}_{n}(1 \leq n \leq \infty)$. Then there is a subgroup $H \leq \mathbb{F}_{n}$ of infinite index such that the generalized shift action $s_{\Gamma, \Gamma / H}$ of $\Gamma$ on $2^{\Gamma / H}$ is maximum in the order $\prec$ of weak containment.

A similar result holds for representations: For $\Gamma=\mathbb{F}_{n}$, there is an infinite index subgroup $H \leq \Gamma$ such that the quasi-regular representation $\lambda_{\Gamma / H}$ of $\Gamma$ on $\ell^{2}(\Gamma / H)$ weakly contains every unitary representation of $\Gamma$.

It follows that for such $H$ the action of $\Gamma=\mathbb{F}_{n}$ on $I=\Gamma / H$ is transitive, faithful and amenable. (Amenability means that $1_{\Gamma} \prec \lambda_{\Gamma / H}$.) The existence of such actions was first proved by van Douwen and later other examples were found by Glasner-Monod and Grigorchuk-Nekrashevych (see GlasnerMonod [GM] for more details). The above result provides an alternative such construction, which has the additional property that $\pi \prec \lambda_{\Gamma / H}$ for every unitary representation of $\Gamma=\mathbb{F}_{n}$ (instead of just $1_{\Gamma} \prec \lambda_{\Gamma / H}$ ). Moreover, for $n \geq 2$, one can find such $H$ for which in addition $\lambda_{\Gamma / H}$ is irreducible. The existence of irreducible representations of $\mathbb{F}_{n}$ that weakly contain any unitary representation was originally proved in Yoshizawa [Y]. (For another proof see Kechris [Ke09], Appendix H, (C).)

(D) This paper is organized as follows. Section 1 reviews various preliminaries and establishes general notation. In Section 2 we discuss actions on trees and in Section 3 we prove Theorem 1 (see Theorem 3.1) and discuss the application to costs. In Section 4 we discuss the properties EMD and MD and some related facts and questions. Section 5 contains the proof of Theorem 7 (see Theorem 5.1). In Section 6 we discuss various results concerning density and meagerness conditions for various sets of actions (both within the space of all actions and also within the space of all actions "included" 
in the full group of an equivalence relation). Finally, there is an appendix reviewing the concept of co-induced action and some facts concerning this notion that are used in the paper.

Acknowledgements. I would like to thank M. Abert, D. Gaboriau, A. Ioana, N. Monod, Y. Shalom and T. Tsankov for many useful discussions or comments concerning this paper and G. Hjorth for allowing me to include 6.3 below. The research of the author was partially supported by NSF Grant DMS-0455285.

\section{Preliminaries}

(A) We work throughout in standard Borel spaces $X$, i.e., sets equipped with a $\sigma$-algebra of subsets (called Borel sets), which is isomorphic to the $\sigma$-algebra of Borel sets on a Polish (separable, completely metrizable) space. A (Borel) measure $\mu$ on such a space $X$ is a measure on the $\sigma$-algebra of Borel sets. It is a probability measure if $\mu(X)=1$. Unless otherwise indicated, we consider only probability measures in the sequel.

If $a: G \times S \rightarrow S$ is an action of a group $G$ on a set $S$, we let

$$
g^{a}(s)=a(g, s)
$$

for $g \in G, s \in S$. We also put

$$
g \cdot s=a(g, s)
$$

if there is no danger of confusion. Given a countable (discrete) group $\Gamma$, a Borel action of $\Gamma$ on $(X, \mu)$ is measure preserving if $\mu(\gamma \cdot A)=\mu(A)$, for every $\gamma \in \Gamma$ and Borel $A \subseteq X$.

We let

$$
A(\Gamma, X, \mu)
$$

be the space of all measure preserving actions of $\Gamma$ on $(X, \mu)$, where the actions $a, b$ are identified if $\gamma^{a}=\gamma^{b}$, $\mu$-a.e., $\forall \gamma \in A$. An action $a \in A(\Gamma, X, \mu)$ is free if $\forall \gamma \neq 1(\gamma \cdot x \neq x, \mu$-a.e. $)$. We denote by

$$
\operatorname{FR}(\Gamma, X, \mu)
$$

the set of free actions. An action $a \in A(\Gamma, X, \mu)$ is ergodic if there are no non-trivial invariant sets. Again we denote by

$$
\operatorname{ERG}(\Gamma, X, \mu)
$$


the set of such actions.

If $a_{i} \in A\left(\Gamma, X_{i}, \mu_{i}\right), i \in I$, are actions, where $I$ is countable, the (diagonal) product action

$$
\prod_{i \in I} a_{i} \in A\left(\Gamma, X^{I}, \mu^{I}\right)
$$

is defined by

$$
\gamma \cdot\left(x_{i}\right)_{i \in I}=\left(\gamma \cdot x_{i}\right)_{i \in I}
$$

A particular case is the product

$$
a \times b
$$

of two actions.

(B) We use the following notation for some particular actions:

(a) If $\Gamma \leq \Delta$ are countable groups, $a_{\Delta / \Gamma}$ is the canonical action of $\Delta$ on $\Delta / \Gamma(=$ the set of left cosets of $\Gamma$ in $\Delta)$ :

$$
\delta \cdot \delta^{\prime} \Gamma=\delta \delta^{\prime} \Gamma
$$

For any set $X, s_{\Gamma, X}$ is the shift action of $\Gamma$ on $X^{\Gamma}$ given by

$$
\gamma \cdot f(\delta)=f\left(\gamma^{-1} \delta\right)
$$

When $X=\{0,1\}=2$, we write

$$
s_{\Gamma} \equiv s_{\Gamma, 2} .
$$

If $\Gamma \leq \Delta$, we write $s_{\Delta, \Delta / \Gamma, X}$ for the generalized shift action of $\Delta$ on $X^{\Delta / \Gamma}$ given by

$$
\delta \cdot f\left(\delta^{\prime} \Gamma\right)=f\left(\delta^{-1} \delta^{\prime} \Gamma\right)
$$

Again when $X=2$, we write

$$
s_{\Delta, \Delta / \Gamma} \equiv s_{\Delta, \Delta / \Gamma, 2}
$$

More generally, if $\Gamma$ acts on a countable set $I$, we define the generalized shift action of $s_{\Gamma, I, X}$ on $X^{I}$ by

$$
\delta \cdot f(i)=f\left(\delta^{-1} \cdot i\right)
$$

and again we let $s_{\Gamma, I} \equiv s_{\Gamma, I, 2}$. 
(C) We denote by

$$
\operatorname{Aut}(X, \mu)
$$

the group of measure preserving automorphisms of $(X, \mu)$, where two such automorphisms $S, T$ are identified if $S=T$, $\mu$-a.e. Thus $A(\Gamma, X, \mu)$ can be viewed as the set of all homomorphisms of $\Gamma$ into $\operatorname{Aut}(X, \mu)$.

The group $\operatorname{Aut}(X, \mu)$ has two canonical topologies, the weak and the uniform. The weak topology is the topology generated by the maps

$$
T \mapsto T(A),
$$

where $T \in \operatorname{Aut}(X, \mu), A \in \operatorname{MALG}(X, \mu)=$ the measure algebra of $(X, \mu)$, and $\operatorname{MALG}(X, \mu)$ is equipped with the metric $d_{\mu}(A, B)=\mu(A \Delta B)$ (and the corresponding topology). The group $\operatorname{Aut}(X, \mu)$ is a Polish group in the weak topology.

The uniform topology on $\operatorname{Aut}(X, \mu)$ is the one induced by the following complete metric

$$
\delta_{\mu}(S, T)=\mu(\{x: S(x) \neq T(x)\}) .
$$

It is not separable, if $\mu$ is non-atomic.

We equip the product space $\operatorname{Aut}(X, \mu)^{\Gamma}$ with the product topology (in either one of the two topologies on $\operatorname{Aut}(X, \mu))$. Then $A(\Gamma, X, \mu) \subseteq \operatorname{Aut}(X, \mu)^{\Gamma}$ is a closed subspace (in either one of these topologies), and we equip it with the relative topology called, respectively, the weak and the uniform topology on $A(\Gamma, X, \mu)$. The weak topology is Polish and the uniform topology is completely metrizable.

(D) Two actions $a \in A(\Gamma, X, \mu), b \in A(\Gamma, Y, \nu)$ are isomorphic, in symbols

$$
a \cong b,
$$

if there is an isomorphism $\varphi:(X, \mu) \rightarrow(Y, \nu)$ such the $\varphi \gamma^{a} \varphi^{-1}=\gamma^{b}, \forall \gamma \in \Gamma$. If $(X, \mu)=(Y, \nu)$ and we consider the action of $\operatorname{Aut}(X, \mu)$ on $A(\Gamma, X, \mu)$ by conjugation, $S \cdot a \equiv S a S^{-1}$, where

$$
\gamma^{S a S^{-1}}=S \gamma^{a} S^{-1}, \forall \gamma \in \Gamma,
$$

then $a, b \in A(\Gamma, X, \mu)$ are isomorphic iff they are conjugate.

If $a \in A(\Gamma, X, \mu), b \in A(\Gamma, Y, \nu)$, then $b$ is a factor of $a$, in symbols

$$
b \sqsubseteq a,
$$


if there is a Borel map $\varphi: X \rightarrow Y$ such that $\varphi_{*} \mu=\nu$ (i.e., $\nu(A)=\mu\left(\varphi^{-1}(A)\right)$ and $\varphi\left(\gamma^{a}(x)\right)=\gamma^{b}(\varphi(x)), \forall \gamma \in \Gamma, \mu$-a.e. For example, $a \sqsubseteq a \times b$.

Occasionally we need to talk about continuous actions of $\Gamma$ on compact spaces. In this situation, we say that a continuous action $b$ of $\Gamma$ on $L$ is a factor of a continuous action $a$ of $\Gamma$ on $K$ if there is a continuous map $\varphi: K \rightarrow L$ such that $\varphi\left(\gamma^{a}(x)\right)=\gamma^{b}(\varphi(x))$.

(E) For $a \in A(\Gamma, X, \mu), b \in A(\Gamma, Y, \nu)$ we say that $a$ is weakly contained in $b$, in symbols

$$
a \prec b,
$$

if for any Borel sets $A_{1}, \ldots, A_{n} \subseteq X$, any $F \subseteq \Gamma$ finite, and any $\epsilon>0$, there are $B_{1}, \ldots, B_{n} \subseteq Y$ such that

$$
\left|\mu\left(\gamma^{a}\left(A_{i}\right) \cap A_{j}\right)-\nu\left(\gamma^{b}\left(B_{i}\right) \cap B_{j}\right)\right|<\epsilon,
$$

$\forall \gamma \in F, \forall i, j \leq n$. See Kechris [Ke09], Section 10, (C) for more information about this concept. When $(X, \mu),(Y, \nu)$ are non-atomic, then $a \prec b$ iff $a$ is in the weak closure of the set of isomorphic copies of $b$ in $A(\Gamma, X, \mu)$. In particular, if also $(X, \mu)=(Y, \nu)$, then $a \prec b$ iff $a$ is in the weak closure of the conjugacy class of $b$. It is easy to verify that

$$
a \sqsubseteq b \Rightarrow a \prec b .
$$

It is also easy to see that $\prec$ is a partial (pre-)order. The associated equivalence relation

$$
a \sim b \Leftrightarrow a \prec b \& b \prec a,
$$

is called weak equivalence.

(F) Given a standard Borel space $X$, an equivalence relation $E$ on $X$ is called countable if every equivalence class $[x]_{E}, x \in X$, is countable. By a result of Feldman-Moore [FM], for every such $E$ there is a Borel action $a$ of $\Gamma$ on $X$ such that

$$
E=E_{a},
$$

where $E_{a}$ is the equivalence relation induced by $a$,

$$
x E_{a} y \Leftrightarrow \exists \gamma \in \Gamma(\gamma \cdot x=y) .
$$

If now $\mu$ is a measure on $X$, then $E$ is measure preserving if $E=E_{a}$, for some action $a \in A(\Gamma, X, \mu)$ (this notion is independent of the action that induces $E)$. 
For a countable, measure preserving $E$ on $(X, \mu)$ we let

$$
[E]=\{T \in \operatorname{Aut}(X, \mu): T(x) E x, \mu \text {-a.e. }\}
$$

be the full group of $E$. It is a closed, separable subgroup of $\operatorname{Aut}(X, \mu)$ in the uniform topology. Letting below $\varphi$ vary over all partial, measure preserving bijections $\varphi: A \rightarrow B$ between Borel subsets of $X$, we let

$$
[[E]]=\{\varphi: \varphi(x) E x, \mu \text {-a.e. }\}
$$

Thus $[E]=[[E]] \cap \operatorname{Aut}(X, \mu)$.

(G) For any countable, measure preserving $E$ on $(X, \mu)$ we denote by

$$
C_{\mu}(E) \equiv C(E)
$$

the cost of $E$ (see Gaboriau [G] or Kechris-Miller [KM]). If $a \in A(\Gamma, X, \mu)$, let

$$
C_{\mu}(a) \equiv C(a)=C\left(E_{a}\right) .
$$

The cost of a group $\Gamma$ is defined by

$$
C(\Gamma)=\inf \left\{C_{\mu}(a): a \in \operatorname{FR}(\Gamma, X, \mu), \mu \text { non-atomic }\right\} .
$$

We say that $\Gamma$ has fixed price if $C_{\mu}(a)=C(\Gamma), \forall a \in \operatorname{FR}(\Gamma, X, \mu), \mu$ nonatomic.

(H) For each (separable) Hilbert space $\mathcal{H}$, we denote by $\operatorname{Rep}(\Gamma, \mathcal{H})$ the space of unitary representations of $\Gamma$ on $\mathcal{H}$, i.e., homomorphisms from $\Gamma$ into $U(\mathcal{H})$, the unitary group of $\mathcal{H}$. We equip $U(\mathcal{H})$ with the weak (equivalently the strong) topology and $\operatorname{Rep}(\Gamma, H)$ with the product topology (viewing $\operatorname{Rep}(\Gamma, \mathcal{H})$ as a closed subspace of $\left.U(\mathcal{H})^{\Gamma}\right)$. As usual, if $\pi \in \operatorname{Rep}\left(\Gamma, \mathcal{H}_{1}\right), \rho \in$ $\operatorname{Rep}\left(\Gamma, \mathcal{H}_{2}\right)$, we let

$$
\pi \prec \rho,
$$

if $\pi$ is weakly contained in $\rho$. We also write

$$
\pi \leq \rho
$$

if $\pi$ is (isomorphic to) a subrepresentation of $\rho$, and let

$$
\pi \cong \rho
$$


if $\pi, \rho$ are isomorphic.

If $\Gamma \leq \Delta$, then we denote by $\lambda_{\Delta / \Gamma}$ the quasi-regular representation of $\Delta$ on $\ell^{2}(\Delta / \Gamma)$ given by

$$
\lambda_{\Delta / \Gamma}(\delta)(f)\left(\delta^{\prime} \Gamma\right)=f\left(\delta^{-1} \delta^{\prime} \Gamma\right) .
$$

Finally $1_{\Gamma}$ is the trivial 1-dimensional representation of $\Gamma$.

For each $a \in A(\Gamma, X, \mu)$, we let $\kappa^{a}$ be the Koopman (unitary) representation associated to $a$, which is the unitary representation of $\Gamma$ on $L^{2}(X, \mu)$ given by

$$
\kappa^{a}(\gamma)(f)(x)=f\left(\gamma^{-1} \cdot x\right)
$$

We also let $\kappa_{0}^{a}$ be the restriction of $\kappa^{a}$ to the orthogonal of the constant functions in $L^{2}(X, \mu)$. It can be shown that if $a, b \in A(\Gamma, X, \mu), \mu$ non-atomic, then

$$
a \prec b \Rightarrow \kappa^{a} \prec \kappa^{b}, \kappa_{0}^{a} \prec \kappa_{0}^{b},
$$

(see [Ke09], Section 10, (C)).

\section{(I) Conventions}

(a) Throughout the paper, when we work in a measure theoretic context, we neglect null sets, unless there is a danger of confusion. In particular, we do not distinguish between saying that a certain property of $x \in X$, where $(X, \mu)$ is a measure space, is true for all $x$ or for $\mu$-almost all $x$.

(b) The measure spaces $(X, \mu)$ in the sequel will be always assumed to be non-atomic, unless otherwise explicitly stated or obviously understood from the context (e.g., when $X$ is finite).

\section{Actions on trees}

For our purposes in this paper, a tree is an acyclic, connected (simple, undirected) rooted graph $T=\left(V, E, v_{0}\right)$ with vertex set $V$, edge set $E$ and root $v_{0} \in V$. For every $v \in V$ there is a unique path $v_{0}, v_{1}, \ldots, v_{n}=v$ of distinct vertices with $v_{i+1} E v_{i}$ from the root $v_{0}$ to $v$. The children of $v$ are all vertices adjacent to $v$ different from $v_{n-1}$ and the parent of $v$ is $v_{n-1}$. The nth level $(n \geq 0)$ of $T$, in symbols $T_{n}$, consists of all $v \in T$ for which the unique path from $v_{0}$ to $v$ has $n+1$ vertices as above. If $v \in T_{n}$, we write $|v|=n$. (Thus $T_{0}=\left\{v_{0}\right\}$ and $\left|v_{0}\right|=0$.) 
We say that $T$ is finite splitting if every $v \in V$ has finitely many children. A terminal node of $T$ is a vertex $v$ with no children.

From now on we will assume (unless otherwise explicitly indicated) that all trees are finitely splitting and have no terminal nodes. For such a tree $T$, the boundary $\partial T$ of $T$ consists of all infinite sequences $\left(v_{0}, v_{1}, v_{2}, \ldots\right)$ of distinct vertices with $v_{n+1}$ adjacent to $v_{n}$. It is clearly nonempty and compact metrizable in the topology generated by the basic open sets

$$
N_{v}=\left\{\left(v_{0}, v_{1}, \ldots\right) \in \partial T: v=v_{n}\right\},
$$

for $v \in T_{n}, n \geq 0$. (Thus $N_{v_{0}}=\partial T$.) Note that these are actually clopen, so $\partial T$ is 0 -dimensional.

We will consider actions of infinite, countable (discrete) groups $\Gamma$ on trees. An action of $\Gamma$ on $T$ is an action of $\Gamma$ by automorphisms of $T$. In particular, $\Gamma$ fixes $v_{0}$ and acts on each $T_{n}$. Moreover, if $v \in T_{n}, w \in T_{n+1}$ and $w$ is a child of $v$, then $\gamma \cdot v \in T_{n}, \gamma \cdot w \in T_{n+1}$ and $\gamma \cdot w$ is a child of $\gamma \cdot v$. The action of $\Gamma$ induces an action of $\Gamma$ on $\partial T$ via

$$
\gamma \cdot\left(v_{0}, v_{1}, \ldots\right)=\left(\gamma \cdot v_{0}, \gamma \cdot v_{1}, \ldots\right) .
$$

This is clearly an action by homeomorphisms on $\partial T$. Given a probability measure $\mu$ on $\partial T$, let $\mu(v)=\mu\left(N_{v}\right)$. Then $\mu$ is invariant under the $\Gamma$-action iff $\mu(v)=\mu(\gamma \cdot v), \forall \gamma \in \Gamma$.

The action of $\Gamma$ on $T$ is level transitive if $\Gamma$ acts transitively on each $T_{n}$. In this case the associated action of $\Gamma$ on $\partial T$ is uniquely ergodic, i.e., has a unique invariant (thus necessarily ergodic) probability measure $\mu_{T}$ defined by $\mu_{T}(v)=\frac{1}{\operatorname{card}\left(T_{n}\right)}$, for $v \in T_{n}$. We will always consider this measure when we study the $\Gamma$-action on $\partial T$. Finally, a level transitive action is minimal, i.e., every orbit in $\partial T$ is dense.

We now define the orbit tree $O(T)$ associated to the $\Gamma$-action on $T$. The $n$th level of $O(T)$ consists of all the orbits of $\Gamma$ on $T_{n}$. If $o$ is an $n$th level orbit and $o^{\prime}$ an $(n+1)$ th level orbit, then $o^{\prime}$ is a child of $o$ if for any $v^{\prime} \in o^{\prime}$, if $v$ is the parent of $v^{\prime}$, then $v \in o$.

If $e \in \partial(O(T))$, say $e=\left(o_{0}=\left\{v_{0}\right\}, o_{1}, o_{2}, \ldots\right)$, then let $T_{e}$ be the subtree of $T$ determined by

$$
v \in T_{e} \Leftrightarrow v \in o_{n},
$$

for $v \in T_{n}, n \geq 0$. Clearly $T_{e}$ is $\Gamma$-invariant and the action of $\Gamma$ on $T_{e}$ is level transitive. Put $X_{e}=\partial T_{e}$. Then $\left\{X_{e}\right\}_{e \in \partial(O(T))}$ is a decomposition of 
$\partial T$ into closed $\Gamma$-invariant sets on which $\Gamma$ acts minimally and with a unique invariant, ergodic probability measure $\mu_{e}$.

Proposition 2.1 Every $\Gamma$-invariant, probability measure on $\partial T$ is of the form $\mu_{e}$.

Proof. Fix such a measure $\nu$. Now $\partial T=\bigsqcup_{i=1}^{k} X_{o_{i}}$, is a decomposition into $\Gamma$-invariant sets, where $o_{1}, \ldots o_{k}$ are the $\Gamma$-orbits on $T_{1}$, and we let $X_{o}=$ $\bigcup_{v \in o} N_{v}$. Thus there is (a unique) $o_{i}$, say $o_{1}$, such that $\nu\left(X_{o_{1}}\right)=1$. Next $X_{o_{1}}$ decomposes into the $\Gamma$-invariant sets $X_{o_{1}^{\prime}}, \ldots, X_{o_{\ell}^{\prime}}$, where $o_{1}^{\prime}, \ldots o_{\ell}^{\prime}$ are the $\Gamma$-orbits of $T_{2}$ which are children of $o_{1}$. Again there is unique $o^{\prime}$, say $o_{1}^{\prime}$, with $\nu\left(X_{o_{1}^{\prime}}\right)=1$. Proceed this way to define $e=\left(o_{0}=\left\{v_{0}\right\}, o_{1}, o_{1}^{\prime}, o_{1}^{\prime \prime}, \ldots\right) \in$ $\partial(O(T))$. Then $X_{e}=X_{o_{1}} \cap X_{o_{1}^{\prime}} \cap X_{o_{1}^{\prime \prime}} \cap \ldots$, so $\nu\left(X_{e}\right)=1$, and as $\nu$ is $\Gamma$-invariant, $\nu=\mu_{e}$.

Thus

$$
\mathcal{E}=\left\{\mu_{e}: e \in \partial(O(T))\right\}
$$

is the space of all $\Gamma$-invariant, ergodic probability measures on $\partial T$. For $x \in \partial T$, put

$$
\mu_{x}=\mu_{e} \text {, where } x \in X_{e} .
$$

Proposition 2.2 If $\mu$ is a $\Gamma$-invariant probability measure on $\partial T$, then

$$
\mu=\int \mu_{x} d \mu(x)
$$

Proof. It is enough to show for any $v \in V$, that $\mu\left(N_{v}\right)=\int \mu_{x}\left(N_{v}\right) d \mu(x)$. Let $v \in T_{n}$ and let $o$ be the $\Gamma$-orbit of $v$. Then $\mu\left(N_{v}\right)=\frac{\mu\left(X_{o}\right)}{\operatorname{card}(o)}$. Clearly $\mu_{x}\left(N_{v}\right)=0$, unless $x \in X_{o}$, so

$$
\begin{aligned}
\int \mu_{x}\left(N_{v}\right) d \mu(x) & =\int_{X_{o}} \mu_{x}\left(N_{v}\right) d \mu(x) \\
& =\frac{\mu\left(X_{o}\right)}{\operatorname{card}(o)}=\mu\left(N_{v}\right)
\end{aligned}
$$

Thus the map $\pi(x)=\mu_{x}$ has the following properties:

(i) It is a Borel surjection of $\partial T$ onto the standard Borel space $\mathcal{E}$. 
(ii) It is $\Gamma$-invariant.

(iii) For any $\rho \in \mathcal{E}, \pi^{-1}(\{\rho\})$ is $\Gamma$-invariant, $\rho\left(\pi^{-1}(\{\rho\})\right)=1$ and the action of $\Gamma$ on $\pi^{-1}(\{\rho\})$ is uniquely ergodic as witnessed by $\rho$.

(iv) If $\mu$ is a $\Gamma$-invariant probability measure on $\partial T$, then $\mu=\int \pi(x) d \mu(x)$.

Thus $\pi$ is the Ergodic Decomposition of the $\Gamma$-action on $\partial T$ (in the strong sense of Farrell, Varadarajan-see, e.g., Kechris-Miller [KM], 3.3, where the context is that of countable equivalence relations but holds equally well in the context of actions by countable groups).

Now consider a level transitive action of $\Gamma$ on $T$. Fix $x \in \partial T, x=$ $\left(v_{0}, v_{1}, v_{2}, \ldots\right)$ and let $\Gamma_{n}$ be the stabilizer of $v_{n}$. Then $\Gamma_{0}=\Gamma \geq \Gamma_{1} \geq$ $\Gamma_{2} \geq \ldots$ and $\left[\Gamma: \Gamma_{n}\right]<\infty$ (in fact $\left.\left[\Gamma: \Gamma_{n}\right]=\operatorname{card}\left(T_{n}\right)\right)$. We call such a sequence $\left\{\Gamma_{n}\right\}$ a chain in $\Gamma$. Conversely, for any chain $\left\{\Gamma_{n}\right\}$ we can define a tree $T\left(\Gamma,\left\{\Gamma_{n}\right\}\right)$, where the $n$th level $T_{n}=T_{n}\left(\Gamma,\left\{\Gamma_{n}\right\}\right)$ consists of the cosets $g \Gamma_{n}$ of $\Gamma_{n}$, i.e., $T_{n}=\Gamma / \Gamma_{n}$, and $h \Gamma_{n+1}$ if a child of $g \Gamma_{n}$ if $h \Gamma_{n+1} \subseteq g \Gamma_{n}$. The group $\Gamma$ acts on $T\left(\Gamma,\left\{\Gamma_{n}\right\}\right)$ in the obvious way: $\gamma \cdot g \Gamma_{n}=\gamma g \Gamma_{n}$. If $T_{n}=T\left(\Gamma,\left\{\Gamma_{n}\right\}\right)$, clearly $\Gamma_{n} \in T_{n}$ and the stabilizer of $T_{n}$ in the $\Gamma$-action is equal to $\Gamma_{n}$. It is clear that in the particular case that $\Gamma_{n}$ is the stabilizer of $v_{n}$, in the preceding notation, the map $g \Gamma_{n} \mapsto g \cdot v_{n}$ is an isomorphism of the $\Gamma$-action on $T$ and the $\Gamma$-action on $T\left(\Gamma,\left\{\Gamma_{n}\right\}\right)$. Thus all level transitive $T$-action on trees are actions of $\Gamma$ on trees of the from $T\left(\Gamma,\left\{\Gamma_{n}\right\}\right)$.

Now assume that $\Gamma$ is a residually finite group, i.e., $\bigcap\{H \leq \Gamma:[\Gamma: H]<$ $\infty\}=\{1\}$. Equivalently this means that there is a chain $\Gamma=\Gamma_{0} \geq \Gamma_{1} \geq \ldots$, with $\bigcap_{n} \Gamma_{n}=\{1\}$. Such a chain can always be taken to be normal, i.e., $\Gamma_{n} \triangleleft \Gamma$, since any subgroup of finite index contains a normal one with the same property, i.e., the intersection of its conjugates. If $\left\{\Gamma_{n}\right\}$ is a normal chain with $\bigcap_{n} \Gamma_{n}=\{1\}$, then $\partial T\left(\Gamma,\left\{\Gamma_{n}\right\}\right)$ is also a group with multiplication defined as follows: If $x=\left(x_{0}, x_{1}, \ldots\right), y=\left(y_{0}, y_{1}, \ldots\right)$, where $x_{n}, y_{n} \in \Gamma / \Gamma_{n}$, then $x y=\left(x_{0} y_{0}, x_{1} y_{1}, \ldots\right)$. This turns $\partial T\left(\Gamma,\left\{\Gamma_{n}\right\}\right)$ into a compact, metrizable, 0-dimensional topological group. Next note that $\Gamma$ can be identified with a dense subgroup of $\partial T\left(\Gamma,\left\{\Gamma_{n}\right\}\right)$ by identifying $\gamma \in \Gamma$ with $\left(\Gamma, \gamma \Gamma_{1}, \gamma \Gamma_{2}, \ldots\right)$. With this identification the action of $\Gamma$ on $\partial T\left(\Gamma,\left\{\Gamma_{n}\right\}\right)$ is simply the lefttranslation action of $\Gamma$ on the group $\partial T\left(\Gamma,\left\{\Gamma_{n}\right\}\right)$. In particular, it is free.

Consider now the special case of a normal chain $\Gamma=\Gamma_{0} \geq \Gamma_{1} \geq \ldots$ which is cofinal, i.e., $\forall H \leq \Gamma\left([\Gamma: H]<\infty \Rightarrow \exists n, H \geq \Gamma_{n}\right.$ ) (in particular $\left.\bigcap_{n} \Gamma_{n}=\{1\}\right)$. Then the group $\hat{\Gamma}=\partial T\left(\Gamma,\left\{\Gamma_{n}\right\}\right)$ is called the profinite completion of $\Gamma$. It is independent of $\left\{\Gamma_{n}\right\}$ as it is isomorphic to the inverse 
limit given by $\lim _{\longleftarrow}\{\Gamma / N: N \triangleleft \Gamma,[\Gamma: N]<\infty\}$. We denote the action of $\Gamma$ on $\hat{\Gamma}$ by $p_{\Gamma}$. The canonical invariant measure for this action is of course the (normalized) Haar measure on $\hat{\Gamma}$. It is clearly non-atomic, since $\Gamma$ is infinite.

Proposition 2.3 For any chain $\Gamma=\Gamma_{0} \geq \Gamma_{1} \geq \ldots$, the action of $\Gamma$ on $\partial T\left(\Gamma,\left\{\Gamma_{n}\right\}\right)$ is a factor of the action $p_{\Gamma}$, both in the topological and measure theoretic sense.

Proof. Fix a normal cofinal chain $\Gamma=N_{0} \geq N_{1} \geq \ldots$ We will find a surjective continuous map $\pi: \partial T\left(\Gamma,\left\{N_{n}\right\}\right) \rightarrow \partial T\left(\Gamma,\left\{\Gamma_{n}\right\}\right)$ that preserves the action of $\Gamma$.

Let $k_{1}<k_{2}<\ldots$ be such that $k_{1}=$ (least $k$ with $N_{k} \subseteq \Gamma_{1}$ ) and $k_{n+1}=$ (least $k>k_{n}$, with $\left.N_{k} \subseteq \Gamma_{n+1}\right)$. Then $N_{k_{n}} \subseteq \Gamma_{n}$. Given $x \in$ $\partial T\left(\Gamma,\left\{N_{n}\right\}\right), x=\left(\Gamma, g_{1} N_{1}, g_{2} N_{2}, \ldots\right)$, let $\pi(x)=\left(\Gamma, g_{k_{1}} \Gamma_{1}, g_{k_{2}} \Gamma_{2}, \ldots\right)$. Note that $g_{k_{n}} \Gamma_{n}$ is the unique $\Gamma_{n}$-coset containing the $N_{k_{n}}$-coset $g_{k_{n}} N_{k_{n}}$. Clearly $\pi$ is continuous and preserves the $\Gamma$-actions.

We will next verify that it is surjective. Fix $y=\left(\Gamma, g_{1} \Gamma_{1}, g_{2} \Gamma_{2}, \ldots\right) \in$ $\partial T\left(\Gamma,\left\{\Gamma_{n}\right\}\right)$. Call a sequence $s=\left(\Gamma, h_{1} N_{k_{1}}, \ldots, h_{n} N_{k_{n}}\right)$ good if $h_{1} N_{k_{1}} \supseteq$ $\cdots \supseteq h_{n} N_{k_{n}}$ and $h_{i} N_{k_{i}} \subseteq g_{i} \Gamma_{i}, 1 \leq i \leq n$. Clearly every good $s$ as above has only finitely many good extensions $\left(\Gamma, h_{1} N_{k_{1}}, \ldots, h_{n} N_{k_{n}}, h_{n+1} N_{k_{n+1}}\right)$. We now claim that for each $n$, there is some good $s=\left(\Gamma, h_{1} N_{k_{1}}, \ldots, h_{n} N_{k_{n}}\right)$. Indeed, consider $g_{n} \Gamma_{n}$, and let $h_{n}=g_{n}$, so that $h_{n} N_{k_{n}} \subseteq g_{n} \Gamma_{n}$. Then let $h_{i} N_{k_{i}}$ be the unique coset containing $h_{n} N_{k_{n}}(1 \leq i<n)$. We will show that $h_{i} N_{k_{i}} \subseteq g_{i} \Gamma_{i}$. We have $h_{n} N_{k_{n}} \subseteq h_{i} N_{k_{i}} \cap g_{n} \Gamma_{n} \subseteq h_{i} N_{k_{i}} \cap g_{i} \Gamma_{i}$, so $h_{i} N_{k_{i}} \cap g_{i} \Gamma_{i} \neq \emptyset$, thus $h_{i} N_{k_{i}} \subseteq g_{i} \Gamma_{i}$.

So, by König's Lemma, there is an infinite sequence $\left(\Gamma, h_{1} N_{k_{1}}, h_{2} N_{k_{2}}, \ldots\right)$ such that for each $i, h_{i+1} N_{k_{i+1}} \subseteq h_{i} N_{k_{i}} \subseteq g_{i} \Gamma_{i}$. Let $x=\left(\Gamma, g_{1} N_{1}, g_{2} N_{2}, \ldots\right)$ be the unique element of $\partial T\left(\Gamma,\left\{N_{n}\right\}\right)$ such that $g_{k_{i}} N_{k_{i}}=h_{i} N_{k_{i}}, i=1,2, \ldots$. Clearly then $\pi(x)=y$.

If $\mu$ is the unique invariant probability measure for $p_{\Gamma}$, then $\pi_{*} \mu$ is invariant for the $\Gamma$-action on $\partial T\left(\Gamma,\left\{\Gamma_{n}\right\}\right)$, so it is equal to the unique $\Gamma$-invariant probability measure on $\partial T\left(\Gamma,\left\{\Gamma_{n}\right\}\right)$. Thus $\pi$ preserves the measures as well.

Thus for any level transitive action of $\Gamma$ on a tree $T$, the corresponding action $a$ of $\Gamma$ on $\partial T$ is a factor of $p_{\Gamma}$. In particular, $a$ is weakly contained in $p_{\Gamma}, a \prec p_{\Gamma}$, i.e., $p_{\Gamma}$ is the maximum, in the sense of weak containment, level transitive action of $\Gamma$ on the boundary of a tree. 
A finitely modular action or profinite action of $\Gamma$ on a standard Borel space $X$ is a Borel action for which there is a decreasing sequence of finite Borel partitions $\{X\}=\mathcal{P}_{0} \geq \mathcal{P}_{1} \geq$ (i.e., $\mathcal{P}_{n+1}$ refines $\mathcal{P}_{n}$ ) such that each $\mathcal{P}_{n}$ is $\Gamma$-invariant (setwise) and $\left\{\mathcal{P}_{n}\right\}$ separate points. (See Hjorth [Hj02] or Kechris [Ke07]). If moreover for any $A_{i} \in \mathcal{P}_{i}$ with $A_{0} \supseteq A_{1} \supseteq \ldots$, we have that $\bigcap_{n} A_{n}$ is a singleton, we call this a special modular action (thus special modular $\Rightarrow$ finitely modular).

If $\Gamma$ is an action on a tree $T$ and $\mathcal{P}_{n}=\left\{N_{v}: v \in T_{n}\right\}$, clearly $\left\{\mathcal{P}_{n}\right\}$ shows that the action of $\Gamma$ on $\partial T$ is a special modular action. Conversely, if we have a special modular action of $\Gamma$ on $X$ with witness $\left\{\mathcal{P}_{n}\right\}$, consider the tree $T_{\left\{\mathcal{P}_{n}\right\}}$ whose $n$th level is equal to $\mathcal{P}_{n}$ and $B \in \mathcal{P}_{n+1}$ is a child of $A \in \mathcal{P}_{n}$ if $B \subseteq A$. Then $\Gamma$ acts on $T_{\left\{\mathcal{P}_{n}\right\}}$ and it is clear that the action of $\Gamma$ on $X$ is Borel isomorphic to the action of $\Gamma$ on $\partial T_{\left\{\mathcal{P}_{n}\right\}}$ via the map $x \in X \mapsto\left(A_{0}=X, A_{1}, A_{2}, \ldots\right)$, where $x \in A_{n} \in \mathcal{P}_{n}$.

Even if a finitely modular action of $\Gamma$ on $X$ is not special, the map $x \in X \mapsto\left(A_{0}=X, A_{1}, A_{2}, \ldots\right)$ as above gives a Borel embedding $\pi$ of the $\Gamma$ action on $X$ into the $\Gamma$-action on $\partial T_{\left\{\mathcal{P}_{n}\right\}}$ and thus if $\mu$ is a $\Gamma$-invariant measure on $X$, then $\nu=\pi_{*} \mu$ is a $\Gamma$-invariant measure on $\partial T_{\left\{\mathcal{P}_{n}\right\}}$ and the $\Gamma$-action on $(X, \mu)$ is (measure theoretically) isomorphic to the $\Gamma$-action on $\left(\partial T_{\left\{\mathcal{P}_{n}\right\}}, \nu\right)$. In other words, up to isomorphism, the measure preserving finitely modular actions are the same as the measure preserving actions induced on the boundaries of trees. The ergodic, finitely modular actions correspond to level transitive actions.

Consider now a sequence of actions of $\Gamma$ on trees $T^{1}, T^{2}, \ldots$, the corresponding actions of $\Gamma$ on $\partial T^{1}, \partial T^{2}, \ldots$ and the product action of $\Gamma$ on $\prod_{m=1}^{\infty} \partial T^{m}$ :

$$
\gamma \cdot\left(x^{1}, x^{2}, \ldots\right)=\left(\gamma \cdot x^{1}, \gamma \cdot x^{2}, \ldots\right) .
$$

Proposition 2.4 Given actions of $\Gamma$ on trees $T^{1}, T^{2}, \ldots$, there is a tree $T$ and an action of $\Gamma$ on $T$ such that the $\Gamma$-action on $\partial T$ is Borel isomorphic to the product action of $\Gamma$ on $\prod_{m=1}^{\infty} \partial T^{m}$.

Proof. It is enough to show that the action of $\Gamma$ on $\prod_{m=1}^{\infty} \partial T^{m}$ is a special modular action. Let $\mathcal{P}_{n}$ be the partition of $\prod_{m=1}^{\infty} \partial T^{m}$ given by the clopen sets

$$
\begin{aligned}
R_{v_{1}, \ldots, v_{n}}= & \left\{\left(x_{m}\right) \in \prod_{m=1}^{\infty} \partial T^{m}:\right. \\
& \left.x_{m}(n)=v_{m}, 1 \leq m \leq n\right\},
\end{aligned}
$$


where $\left(v_{1}, v_{2}, \ldots, v_{n}\right) \in \prod_{m=1}^{n}\left(T^{m}\right)_{n}$. Clearly $\left\{\mathcal{P}_{n}\right\}$ witnesses that the product action is a special modular action.

Actually, since the sets $R_{v_{1} \ldots v_{m}}$ are clopen, this argument shows that the action of $\Gamma$ on $\prod_{m=1}^{\infty} \partial T^{m}$ is isomorphic to the action of $\Gamma$ on $\partial T_{\left\{\mathcal{P}_{n}\right\}}$ via a homeomorphism of the two spaces.

\section{Maximality of the profinite action of $\mathbb{F}_{n}$}

It is a general fact, proved independently by Glasner-Thouvenot-Weiss [GTW] and Hjorth (unpublished) (see also Kechris [Ke09], 10.7), that for each countable group $\Gamma$, the space $A(\Gamma, X, \mu)$ has a weakly dense conjugacy class, which in the language of weak containment can be stated as the existence of a maximum (unique up to weak equivalence) action $a_{\infty}$ in the order $\prec$ of weak containment:

$$
\exists a_{\infty} \in A(\Gamma, X, \mu) \forall b \in A(\Gamma, X, \mu)\left(b \prec a_{\infty}\right) .
$$

Such an $a_{\infty}$ can always assumed to be free (by replacing it if necessary by $a_{\infty} \times a$ for any free action $a$ ).

In Kechris [Ke09], 10.13, it is shown that for infinite, finitely generated $\Gamma$, the cost function

$$
a \in \operatorname{FR}(\Gamma, X, \mu) \mapsto C(a),
$$

on the space of free actions with the weak topology, is upper semicontinuous. Since clearly $C$ is invariant under conjugacy, it follows that for any $a, b \in$ $\operatorname{FR}(\Gamma, X, \mu)$, we have

$$
b \prec a \Rightarrow C(b) \geq C(a)
$$

(see Kechris [Ke09], 10.14) and thus for any free $b$,

$$
C\left(a_{\infty}\right) \leq C(b)
$$

and therefore

$$
C\left(a_{\infty}\right)=C(\Gamma)=\text { the cost of } \Gamma .
$$

It is therefore of interest to be able to explicitly "compute" $a_{\infty}$ for various groups $\Gamma$. We will do that below for $\Gamma=\mathbb{F}_{n}$, the free group with $n$ generators $(1 \leq n \leq \infty)$.

Since $\mathbb{F}_{n}(1 \leq n \leq \infty)$ is a residually finite group, let $p_{\mathbb{F}_{n}}$ be the canonical action of $\mathbb{F}_{n}$ on its profinite completion (see $\S 2$ ). 
Theorem 3.1 Let $1 \leq n \leq \infty$. The action $p_{\mathbb{F}_{n}}$ is maximum in the order $\prec$ of weak containment of actions of $\mathbb{F}_{n}$.

Proof. We consider for notational simplicity the case $n<\infty$, the argument in the other case being similar. We can work with $X=2^{\mathbb{N}}, \mu$ the usual product measure. Clearly $A\left(\mathbb{F}_{n}, 2^{\mathbb{N}}, \mu\right)$ can be identified with $\left(\operatorname{Aut}\left(2^{\mathbb{N}}, \mu\right)\right)^{n}$, where $\operatorname{Aut}\left(2^{\mathbb{N}}, \mu\right)$ is the group of measure preserving automorphisms of $\left(2^{\mathbb{N}}, \mu\right)$ with the weak topology. If $\mathbb{F}_{n}=\left\langle\gamma_{1}, \ldots, \gamma_{n}\right\rangle$, with $\gamma_{i}$ free generators, then $\left(S_{1}, \ldots, S_{n}\right) \in\left(\operatorname{Aut}\left(2^{\mathbb{N}}, \mu\right)\right)^{n}$ is identified with the action $a \in A\left(\mathbb{F}_{n}, 2^{\mathbb{N}}, \mu\right)$ for which $\gamma_{i}^{a}=S_{i}, 1 \leq i \leq n$. We will show that given any $a \in A\left(\mathbb{F}_{n}, 2^{\mathbb{N}}, \mu\right)$ and a weak open nbhd $U$ of $a$, there is an action $\rho$ of $\mathbb{F}_{n}$ on a tree $T$, with $\partial T$ uncountable, which is level transitive, such that if $c$ is the associated action of $\mathbb{F}_{n}$ on the boundary $\partial T$, then $U$ contains an isomorphic copy $b$ of $c$. Then by $\S 2$, we have that $c$, and thus $b$, is a factor of $p_{\mathbb{F}_{n}}$, in particular $b \prec p_{\mathbb{F}_{n}}$. Thus $a$ is the weak limit of actions $b \prec p_{\mathbb{F}_{n}}$, and so $a \prec p_{\mathbb{F}_{n}}$.

Fix an automorphism $\sigma$ of the binary tree $2^{<\mathbb{N}}$ which is level transitive and denote by $S$ the corresponding automorphism of $\partial 2^{<\mathbb{N}}=2^{\mathbb{N}}$ (e.g., $S$ could be the odometer). Identify the action $a$ with $\left(S_{1}, \ldots, S_{n}\right)$, where $\gamma_{i}^{a}=S_{i}$. Then we can assume that $U=U_{1} \times \cdots \times U_{n}$, where $U_{1}, \ldots, U_{n}$ are weak open nbhds of $S_{1}, \ldots, S_{n}$, resp., in $\operatorname{Aut}\left(2^{\mathbb{N}}, \mu\right)$.

By the Conjugacy Lemma (see, e.g., Kechris [Ke09], 2.4) there is $P \in$ Aut $\left(2^{\mathbb{N}}, \mu\right)$ with $P^{-1} S P \in U_{1}$. Consider now $P S_{2} P^{-1}, \ldots, P S_{n} P^{-1}$ and their open nbhds $P U_{2} P^{-1}, \ldots, P U_{n} P^{-1}$. By the Weak Approximation Theorem (see, e.g., Kechris [Ke09], 2.1), there is large enough $N$ and permutations $\pi_{2}, \ldots, \pi_{n}$ of $2^{N}$ (the set of binary sequences of length $N$ ) such that if $S_{\pi_{i}}$ on $2^{\mathbb{N}}$ is defined by $S_{\pi_{i}}(\hat{s} x)=\pi_{i}(s)^{\wedge} x$, for $s \in 2^{N}, x \in 2^{\mathbb{N}}$, then $S_{n_{i}} \in P U_{i} P^{-1}, 2 \leq i \leq n$. Consider now the action $d$ of $\mathbb{F}_{n}$ given by $\left(S, S_{\pi_{2}}, \ldots, S_{\pi_{n}}\right)$.

Let $T$ be the tree consisting of all finite sequences $s=\left(s_{0}, s_{1}, \ldots, s_{n-1}\right)$ (including the empty sequence), where $s_{0} \in 2^{N}, s_{i} \in\{0,1\}$, if $1 \leq i<n$. The root of $T$ is the empty sequence and the children of $s$ are all sequences $t=\left(s_{0}, s_{1}, \ldots, s_{n-1}, s_{n}\right), s_{n} \in\{0,1\}$. We can define an action $\rho$ of $\mathbb{F}_{n}$ on $T$ as follows: $\gamma_{1}$ acts as $\sigma$ does; $\gamma_{2}, \ldots, \gamma_{n}$ act via $\gamma_{i} \cdot\left(s_{0}, s_{1}, \ldots, s_{n-1}\right)=$ $\left.\pi_{i}\left(s_{0}\right), s_{1}, \ldots, s_{n-1}\right)$. Since $\sigma$ is level transitive, so is $\rho$. Let $c$ be the associated action on $\partial T$. Clearly $c$ is isomorphic to $d$ via the isomorphism $\left(s_{0}, s_{1}, \ldots\right) \in$ $\partial T \mapsto s_{0} \hat{s}_{1}{ }^{\wedge} \ldots$ Now if $b=P^{-1} d P=\left(P^{-1} S P, P^{-1} S_{\pi_{2}} P, \ldots, P^{-2} S_{\pi_{n}} P\right)$, then $b \in U_{1} \times U_{2} \times \cdots \times U_{n}$, thus $U$ contains an isomorphic copy $b$ of $c$.

We now combine the preceding result with the main theorem in Abert- 
Nikolov $[\mathrm{AN}]$ to give a new proof that the cost of $\mathbb{F}_{n}$ is equal to $n(1 \leq n<$ $\infty)$.

Let $\Gamma$ be a finitely generated group and $\Gamma=\Gamma_{0} \geq \Gamma_{1} \geq \ldots$ a chain. Let $T\left(\Gamma,\left\{\Gamma_{n}\right\}\right)$ be the corresponding tree and let $a_{\Gamma,\left\{\Gamma_{n}\right\}}$ the associated action of $\Gamma$ on $\partial T\left(\Gamma,\left\{\Gamma_{n}\right\}\right)$. Note that if each $\Gamma_{n}$ is normal and $\bigcap_{n} \Gamma_{n}=\{1\}$, then $a_{\Gamma,\left\{\Gamma_{n}\right\}}$ is free.

Theorem 3.2 (Abert-Nikolov $[\mathrm{AN}]$ ) If the action $a_{\Gamma,\left\{\Gamma_{n}\right\}}$ is free, then

$$
C\left(a_{\Gamma,\left\{\Gamma_{n}\right\}}\right)=\lim _{n \rightarrow \infty} \frac{d\left(\Gamma_{n}\right)-1}{\left[\Gamma: \Gamma_{n}\right]}+1,
$$

where $d\left(\Gamma_{n}\right)=\operatorname{rank}\left(\Gamma_{n}\right)=$ minimum number of generators of $\Gamma_{n}$.

Remark. If $\Gamma$ is finitely generated and $\Delta \leq \Gamma$ has finite index, then $d(\Delta)-1 \leq[\Gamma: \Delta](d(\Gamma)-1)$, with equality if $\Gamma$ is free (see, Lyndon-Schupp [LS]). So if $H \leq \Delta \leq \Gamma$ have finite index, $\frac{d(H)-1}{[\Gamma: H]} \leq \frac{d(\Delta)-1}{[\Gamma: \Delta]}$, thus the sequence $\frac{d\left(\Gamma_{n}\right)-1}{\left[\Gamma: \Gamma_{n}\right]}$ is decreasing, and the above limit exists.

Now for $\Gamma=\mathbb{F}_{n}$ and any $H \leq \mathbb{F}_{n}$ of finite index, $\frac{d(H)-1}{[\Gamma: H]}=d\left(\mathbb{F}_{n}\right)-1=$ $n-1$. Therefore, since $p_{\Gamma}$ is the action $a_{\Gamma,\left\{\Gamma_{n}\right\}}$, where $\Gamma_{n}$ is a decreasing sequence of normal, finite index subgroups which is cofinal, we have that $C\left(p_{\mathbb{F}_{n}}\right)=n-1$. Moreover, for any action $a$ of $\mathbb{F}_{n}, C(a) \leq n$, so for any free action $a$ of $\mathbb{F}_{n}, C(a)=n$. Thus we have shown:

Corollary 3.3 (Gaboriau [G]) The cost of $\mathbb{F}_{n}(1 \leq n<\infty)$ is equal to $n$ and $\mathbb{F}_{n}$ has fixed price.

Remark. In a recent paper, Abert and Weiss [AW] have shown that if for an infinite group $\Gamma$, we let $s_{\Gamma}$ be the shift action of $\Gamma$ on $2^{\Gamma}$ (with the usual product measure), then $s_{\Gamma}$ is weakly contained in any free action of $\Gamma$ :

$$
\forall b \in \operatorname{FR}(\Gamma, X, \mu)\left(s_{\Gamma} \prec b\right) .
$$

Thus among the free actions of $\Gamma$ there is a minimum in the order $\prec$. It follows that $C\left(s_{\Gamma}\right) \geq C(b), \forall b \in \mathrm{FR}(\Gamma, X, \mu)$, i.e., $s_{\Gamma}$ realizes the maximum cost of a free action of $\Gamma$.

Note now that there is a minimum, in the order $\prec$ of weak containment, action in $A(\Gamma, X, \mu)$ (where we now consider arbitrary, not necessarily free, actions) iff the group $\Gamma$ is amenable. Indeed, if $a \in A(\Gamma, X, \mu)$ is minimum in 
$\prec$, and $i_{\Gamma} \in A(\Gamma, X, \mu)$ is the trivial action of $\Gamma$, then $a \prec i_{\Gamma}$, so $a=i_{\Gamma}$, i.e., $i_{\Gamma}$ is minimum. Then $i_{\Gamma} \prec s_{\Gamma}$, so (see Kechris [Ke09], 13.2) $\Gamma$ is amenable. Conversely, if $\Gamma$ is amenable, then $i_{\Gamma} \prec a$, for every ergodic $a \in A(\Gamma, X, \mu)$; see Kechris [Ke09], 10.6. Using the ergodic decomposition, this implies that $i_{\Gamma} \prec a$ for every $a \in A(\Gamma, X, \mu)$, so $i_{\Gamma}$ is minimum.

In particular, since, for $\Gamma$ amenable, $s_{\Gamma}$ weakly contains any action (see Kechris [Ke09], 13.2), it follows that for amenable $\Gamma, i_{\Gamma}$ is the minimum and $s_{\Gamma}$ the maximum in the order $\prec$ of weak containment in $A(\Gamma, X, \mu)$ and by Abert-Weiss so is every free action of $\Gamma$.

\section{Density conditions in the space of actions}

It is unclear for what infinite, residually finite groups $\Gamma$ the analog of 3.1 goes through, i.e., the action $p_{\Gamma}$ is maximum in the order $\prec$. Similarly, in the finitely generated case, concerning the (weaker) condition that $C\left(p_{\Gamma}\right)=C(\Gamma)$. Recall that by Abert-Nikolov [AN], for finitely generated such $\Gamma, C\left(p_{\Gamma}\right)=$

$\lim _{n \rightarrow \infty} \frac{d\left(\Gamma_{n}\right)-1}{\left[\Gamma: \Gamma_{n}\right]}+1=\operatorname{RG}(\Gamma)+1$, where $\left\{\Gamma_{n}\right\}$ is a decreasing sequence of normal finite index subgroups which is cofinal, and $\mathrm{RG}(\Gamma)$ is the absolute rank gradient of $\Gamma$,

$$
\mathrm{RG}(\Gamma)=\inf _{H} \frac{d(H)-1}{[\Gamma: H]},
$$

the inf taken over all finite index $H \leq \Gamma$.

Denote by $i_{\Gamma} \times p_{\Gamma}$ the product of the trivial action $i_{\Gamma}$ with $p_{\Gamma}$. This is an action whose ergodic decomposition consists of continuum many copies of $p_{\Gamma}$ and thus (see Kechris-Miller $[\mathrm{KM}], 10.6) C\left(i_{\Gamma} \times p_{\Gamma}\right)=C\left(p_{\Gamma}\right)$. So if $i_{\Gamma} \times p_{\Gamma}$ is maximum in the order $\prec$, we also have $C\left(p_{\Gamma}\right)=C(\Gamma)$, so it is also worth considering the (weaker) condition that $i_{\Gamma} \times p_{\Gamma}$ is such a maximum.

We will provide below some equivalent reformulations of these maximality conditions, relate them to some other properties considered in the literature, discuss some closure properties and raise some questions.

Definition 4.1 Let $\Gamma$ be an infinite, residually finite group. We say that $\Gamma$ has the property EMD if the ergodic, finitely modular actions $a \in A(\Gamma, X, \mu)$ are weakly dense in $A(\Gamma, X, \mu)$.

Recall from $\S 2$ that the ergodic, finitely modular actions in $A(\Gamma, X, \mu)$, up to isomorphism, can be viewed as actions on boundaries of trees on which the 
group acts in a level transitive way. Again by $\S 2$, up to isomorphism, these can be viewed as actions on $\partial T\left(\Gamma,\left\{\Gamma_{n}\right\}\right)$, for some chain $\Gamma=\Gamma_{0} \geq \Gamma_{1} \geq \ldots$, which is proper in the sense that the $\Gamma_{n}$ do not eventually stabilize. (Recall here our convention that $A(\Gamma, X, \mu)$ always denotes the space of measure preserving actions of $\Gamma$ on a non-atomic space $(X, \mu)$, thus $\partial T\left(\Gamma,\left\{\Gamma_{n}\right\}\right)$ is required to be uncountable, which means that $\left\{\Gamma_{n}\right\}$ is proper.)

Proposition 4.2 Let $\Gamma$ be an infinite, residually finite group. Then $\Gamma$ has property EMD iff $p_{\Gamma}$ is maximum in the order $\prec$ of weak containment.

Proof. If $\Gamma$ has property EMD and $a \in A(\Gamma, X, \mu)$, then in any weakly open nbhd $U$ of $a$ there is an isomorphic copy of an action $b$ of $\Gamma$ on $\partial T\left(\Gamma,\left\{\Gamma_{n}\right\}\right)$, for some proper chain $\Gamma=\Gamma_{0} \geq \Gamma_{1} \geq \ldots$ By $2.3, b \prec p_{\Gamma}$ and thus there is an isomorphic copy of $p_{\Gamma}$ in $U$, so $a \prec p_{\Gamma}$ and $p_{\Gamma}$ is maximum in $\prec$.

Conversely, if $p_{\Gamma}$ is maximum in $\prec$, then the isomorphic copies of $p_{\Gamma}$ are weakly dense in $A(\Gamma, X, \mu)$. But clearly $p_{\Gamma}$ is an ergodic finitely modular action and we are done.

Recall that an infinite, residually finite group $\Gamma$ satisfies property $(\tau)$ if $1_{\Gamma} \nprec \kappa_{0}^{p_{\Gamma}}$, where $\kappa_{0}^{p_{\Gamma}}$ is the Koopman representation of $p_{\Gamma}$, restricted to the orthogonal of the constant functions, and $1_{\Gamma}$ is the trivial 1-dimensional representation.

Proposition 4.3 Let $\Gamma$ be an infinite residually finite group. Then if $\Gamma$ has property EMD, $\Gamma$ does not satisfy property $(\tau)$.

Proof. By 4.2, we have that if $\Gamma$ has property EMD, $p_{\Gamma}$ is maximum in the order $\prec$, so $i_{\Gamma} \prec p_{\Gamma}$. It follows that $1_{\Gamma} \prec \kappa_{0}^{p_{\Gamma}}$ (see Kechris [Ke09], Section 10, (C)), thus $\Gamma$ does not have property $(\tau)$.

Definition 4.4 Let $\Gamma$ be an infinite, residually finite group. Then $\Gamma$ has property EMD* if the ergodic, finitely modular actions are weakly dense in the ergodic actions in $A(\Gamma, X, \mu)$.

Clearly $\mathrm{EMD} \Rightarrow \mathrm{EMD}^{*}$. If $\Gamma$ does not have property $(\mathrm{T})$, then, by a result of Glasner-Weiss (see, e.g., Kechris [Ke09], 12.2), the ergodic actions are dense in $A(\Gamma, X, \mu)$, so EMD $\Leftrightarrow \mathrm{EMD}^{*}$ for such $\Gamma$. The following is proved as in 4.2 . 
Proposition 4.5 Let $\Gamma$ be an infinite, residually finite group. Then $\Gamma$ has property EMD* iff $p_{\Gamma}$ is maximum in the order $\prec$ among the ergodic actions in $A(\Gamma, X, \mu)$.

We will now consider the final weakening of these properties.

Definition 4.6 Let $\Gamma$ be an infinite, residually finite group. Then $\Gamma$ has property $\mathrm{MD}$ if the finitely modular actions are dense in $A(\Gamma, X, \mu)$.

Definition 4.7 An action $a \in A(\Gamma, X, \mu)$ is finite if it factors through a finite group, i.e., there is a finite group $\Delta$, a homomorphism $\pi: \Gamma \rightarrow \Delta$ and an action $b \in A(\Delta, X, \mu)$ such that $\gamma^{a}=\pi(\gamma)^{b}$. Equivalently a is finite if $\left\{\gamma^{a}: \gamma \in \Gamma\right\}$ is a finite subgroup of $\operatorname{Aut}(X, \mu)$.

Proposition 4.8 Let $\Gamma$ be an infinite, residually finite group. Then the following are equivalent:

(i) $\Gamma$ has property MD.

(ii) The finite actions of $\Gamma$ are weakly dense in $A(\Gamma, X, \mu)$.

(iii) The action $i_{\Gamma} \times p_{\Gamma}$ is maximum in the order $\prec$ of weak containment.

Proof. The finite actions are clearly finitely modular, so (ii) $\Rightarrow$ (i).

We next show that (i) $\Rightarrow$ (ii). For that it is enough to show that the finite action are dense in the finitely modular ones. Again for that it is enough to show that if $X=P_{1} \sqcup \cdots \sqcup P_{n}$ is a partition of $X$ into finitely many sets of positive measure and $a$ is an action of $\Gamma$ on $(X, \mu)$ that leaves the partition $\mathcal{P}=\left\{P_{1}, \ldots, P_{n}\right\}$ invariant, then there is a finite action $b$ of $\Gamma$ on $(X, \mu)$ that agrees with $a$ on $\mathcal{P}$, i.e., $\forall \gamma \in \Gamma \forall P \in \mathcal{P}\left(\gamma^{a}(P)=\gamma^{b}(P)\right)$.

It is clear that there is a finite group $\Delta$, an action $c$ of $\Delta$ on $\mathcal{P}$ and a surjective homomorphism $\pi: \Gamma \rightarrow \Delta$ such that for each $P \in \mathcal{P}, \gamma \in \Gamma, \gamma^{a}(P)=$ $\pi(\gamma)^{c}(P)$. (Simply take $\Delta$ to be the group of permutations of $\mathcal{P}$ induced by a.) Next for each $P \in \mathcal{P}, \mu_{P}=\frac{\mu \mid P}{\mu(P)}$ is a non-atomic probability measure on $P$, so fix a measure preserving bijection $\varphi_{P}:\left(P, \mu_{P}\right) \rightarrow([0,1], \lambda)$ (where $\lambda$ is the Lebesgue measure). Put for any $P, Q \in \mathcal{P}, \varphi_{P, Q}=\varphi_{Q}^{-1} \circ \varphi_{P}$. Then $\varphi_{P, Q}:\left(P, \mu_{P}\right) \rightarrow\left(Q, \mu_{Q}\right)$ is a measure preserving bijection and $\varphi_{P, P}=$ id, $\varphi_{P, R}=\varphi_{Q, R} \circ \varphi_{P, Q}$. If $P, Q$ are in the same $\Delta$-orbit, then $\mu(P)=\mu(Q)$, thus $\varphi_{P, Q}:(P, \mu \mid P) \rightarrow(Q, \mu \mid Q)$ is also measure preserving. 
We now define an action $d \in A(\Delta, X, \mu)$ as follows: let $x \in X$ and let $P \in \mathcal{P}$ be such that $x \in P$. Let $\delta^{c}(P)=Q$ and put $\delta^{d}(x)=\varphi_{P, Q}(x)$. From the preceding discussion, it is clear that $d$ is measure preserving and $\delta^{d}(P)=\delta^{c}(P), \forall P \in \mathcal{P}$. Finally, define $b \in A(\Gamma, X, \mu)$ by $\gamma^{b}=\pi(\gamma)^{d}$. Then $\gamma^{b}(P)=\pi(\gamma)^{d}(P)=\pi(\gamma)^{c}(P)=\gamma^{a}(P), \forall P \in \mathcal{P}$, and the proof that $(\mathrm{i}) \Rightarrow$ (ii) is complete.

Since the action $i_{\Gamma} \times p_{\Gamma}$ is finitely modular, it is clear that (iii) $\Rightarrow$ (i). Finally, we show that (i) $\Rightarrow$ (iii).

It is enough to show that if $a \in A(\Gamma, X, \mu)$ is finitely modular, then $a \prec i_{\Gamma} \times p_{\Gamma}$. The action $a$ is isomorphic to a measure preserving action $b$ on the boundary of a tree, thus by $\S 2$ each component in the ergodic decomposition of $b$ is isomorphic to an action of $\Gamma$ on $\partial T\left(\Gamma,\left\{\Gamma_{n}\right\}\right)$ for some chain $\Gamma=\Gamma_{0} \geq \Gamma_{1} \geq \ldots$ By 2.3 , the action of $\Gamma$ on $\partial T\left(\Gamma,\left\{\Gamma_{n}\right\}\right)$ is weakly contained in $p_{\Gamma}$, from which it follows that the action $b$ (and thus the action a) is weakly contained in $i_{\Gamma} \times p_{\Gamma}$.

A similar argument as in the proof of (i) $\Rightarrow$ (iii) in Proposition 4.8, together with 4.5 , shows that

$$
\mathrm{EMD}^{*} \Rightarrow \mathrm{MD}
$$

thus

$$
\mathrm{EMD} \Rightarrow \mathrm{EMD}^{*} \Rightarrow \mathrm{MD}
$$

Moreover, MD implies, for finitely generated $\Gamma$, that $C(\Gamma)=C\left(p_{\Gamma}\right)=\operatorname{RG}(\Gamma)$.

Question 4.9 Does $\mathrm{EMD}^{*} \Rightarrow \mathrm{EMD}$ ?

As we have seen, the answer is positive if $\Gamma$ does not have property $(\mathrm{T})$, so one has the following further problem:

Question 4.10 Does EMD* imply $\neg(\mathrm{T})$ ?

Next we have the question:

Question 4.11 Does $\mathrm{MD} \Rightarrow \mathrm{EMD}^{*}$ ?

A partial answer to these questions is given by the following fact:

Proposition 4.12 Let $\Gamma$ be an infinite, finitely generated, residually finite group that does not have property $(\tau)$. Then $\mathrm{EMD} \Leftrightarrow \mathrm{EMD}^{*} \Leftrightarrow \mathrm{MD}$. 
Proof. It is shown in Abert-Elek [AE], that if $\Gamma$ is as in the statement of the proposition, then $p_{\Gamma} \sim i_{\Gamma} \times p_{\Gamma}$. From this it immediately follows that $\mathrm{MD} \Rightarrow \mathrm{EMD}$.

In view of this, and the fact that $\operatorname{EMD} \Rightarrow \neg(\tau)$, it is clear that the question of whether EMD $\Leftrightarrow$ MD is equivalent to

Question 4.13 Does MD imply $\neg(\tau)$ ?

Note that for infinite, finitely generated, residually finite $\Gamma, \mathrm{MD} \Rightarrow \neg(\tau)$ iff $\left(\mathrm{MD} \Rightarrow \mathrm{EMD}^{*}\right) \&\left(\mathrm{EMD}^{*} \Rightarrow \neg(\mathrm{T})\right)$. It is also unknown whether MD implies $\neg(\mathrm{T})$.

In Lubotzky-Shalom [LSh], the authors say that a residually finite group $\Gamma$ has property FD if the finite unitary representations (i.e., those factoring through a finite quotient of $\Gamma$ ) are dense in the space $\operatorname{Rep}(\Gamma, \mathcal{H})$. Using the fact that any $\pi \in \operatorname{Rep}(\Gamma, \mathcal{H})$ is a subrepresentation of $\kappa_{0}^{a}$ for some $a \in$ $A(\Gamma, X, \mu)$ (see, e.g., Kechris [Ke09], E.1) it follows easily that MD $\Rightarrow$ FD but it is unknown whether FD $\Rightarrow$ MD. In Lubotzky-Zuk [LZ], the question is raised of whether FD $\Rightarrow \neg(\tau)$. Thus 4.13 is an ergodic theory analog of this problem.

In particular, the various examples of groups that fail property FD given, e.g., in Lubotzky-Zuk [LZ], 9.1, also fail property MD.

On the other hand, we have seen in 3.1 that $\mathbb{F}_{n}(1 \leq n \leq \infty)$ has property MD and in fact the property EMD.

We can also see that every infinite, residually finite, amenable group also has property EMD. To see this let $s_{\Gamma}$ be the shift action of $\Gamma$ on $2^{\Gamma}$. Then Abert-Weiss [AW] have shown that $s_{\Gamma} \prec a$ for every free action $a \in$ $A(\Gamma, X, \mu)$. Thus $s_{\Gamma} \prec p_{\Gamma}$. But since $\Gamma$ is amenable, $\forall a \in A(\Gamma, X, \mu)\left(a \prec s_{\Gamma}\right)$ (see Kechris [Ke09], 13.2), so $\forall a \in A(\Gamma, X, \mu)\left(a \prec p_{\Gamma}\right)$. Thus $\Gamma$ has EMD.

We next note some closure properties of the class MD. First, if $\Delta$ is infinite, residually finite with property $\mathrm{MD}$ and $\Gamma \leq \Delta$ is infinite, then $\Gamma$ has property MD. To see this, let $a \in A(\Gamma, X, \mu)$ and let $\operatorname{CInd}_{\Gamma}^{\Delta}(a) \in A(\Delta, Y, \nu)$ be the co-induced action (see the Appendix). By assumption $\operatorname{CIND}_{\Gamma}^{\Delta}(a) \prec$ $i_{\Delta} \times p_{\Delta}$, thus $\operatorname{CIND}_{\Gamma}^{\Delta}(a)\left|\Gamma \prec\left(i_{\Delta} \times p_{\Delta}\right)\right| \Gamma=b$. Clearly $b$ is finitely modular. Moreover $a$ is a factor of $\operatorname{CInd}_{\Gamma}^{\Delta}(a) \mid \Gamma$, so $a \prec \operatorname{CIND}_{\Gamma}^{\Delta}(a) \mid \Gamma \prec b$, thus every action of $\Gamma$ is weakly contained in a finitely modular action, so $\Gamma$ satisfies also MD. It is not clear if the same fact holds for property EMD.

In the opposite direction, if $\Gamma$ is infinite, residually finite with property MD and $\Gamma \leq \Delta$ is such that $[\Delta: \Gamma]<\infty$, then $\Delta$ has also property MD. To 
see this let $b \in A(\Delta, X, \mu)$. Let $a=b \mid \Gamma$ and let $c=\operatorname{Ind}_{\Gamma}^{\Delta}(a)=\operatorname{Ind}_{\Gamma}^{\Delta}(b \mid \Gamma)$ be the induced action (see, e.g., Zimmer [Z] or Kechris [Ke09], Appendix G). By Zimmer [Z], 4.2.22, $c \cong b \times a_{\Delta / \Gamma}$, where $a_{\Delta / \Gamma}$ is the action of $\Delta$ on $\Delta / \Gamma$. In particular $b \prec c$. By assumption $a \prec i_{\Gamma} \times p_{\Gamma}$ and so $b \prec \operatorname{Ind}_{\Gamma}^{\Delta}(a)=$ $c \prec \operatorname{Ind}_{\Gamma}^{\Delta}\left(i_{\Gamma} \times p_{\Gamma}\right)=d$. (We are using here that inducing preserves weak containment of actions, which can be verified as in Appendix A.1.) It is easy to see that $d$ is finitely modular and this shows that $\Delta$ also has property MD. Since the induced action of an ergodic action is ergodic the same argument works as well for the property EMD. In particular, the group $\mathrm{SL}_{2}(\mathbb{Z})$ and all groups of the form $A * B$, where $A, B$ are finite non-trivial groups, have EMD.

In Lubotzky-Shalom [LSh] (see also Lubotzky-Zuk [LZ], 9.11 and 9.12), it is shown that, in certain situations, if $\Gamma \triangleleft \Delta$ and $\Gamma$ has property FD, so does $\Delta$. This is used to provide additional examples of groups with property FD. One would like to prove a similar result for the property MD. The proof of the Lubotzky-Shalom result uses the fact that if $\Gamma \leq \Delta$, the action of $\Delta$ on $\Delta / \Gamma$ is amenable (i.e., admits a finitely additive invariant probability measure) and $\pi$ is a unitary representation of $\Delta$, then $\pi$ is weakly contained in the induced representation $\operatorname{Ind}_{\Gamma}^{\Delta}(\pi \mid \Gamma)$ of the restriction of $\pi$ to $\Gamma$. To prove the analog for the property $\mathrm{MD}$, one would like to have the corresponding result for co-induced actions, but this appears to be unknown - see Problem A.4. Let us state the result about MD that one would like to prove and its implications and then show that an affirmative action to A.4 would indeed provide a proof.

Conjecture 4.14 Let $\Gamma$ be an infinite, finitely generated, residually finite group satisfying MD. Let $\Gamma \triangleleft \Delta$ with $\Delta$ residually finite. Assume that:

(i) For every $N \triangleleft \Gamma$ with $[\Gamma: N]<\infty$, there is $M \triangleleft \Delta$ such that $M \subseteq N$ and $[\Gamma: M]<\infty$.

(ii) $\Delta / \Gamma$ is a residually finite, amenable group.

Then $\Delta$ satisfies MD.

The result of Lubotzky-Shalom [LSh] is that this holds if MD is replaced by FD.

If the Conjecture 4.14 has a positive answer, it would produce the following additional examples of groups with property MD, since Lubotzky-Shalom 
[LSh] and Lubotzky-Zuk [LZ], 9.2., verify that the groups below satisfy the conditions of 4.14.

(a) $H \ltimes \mathbb{F}_{n}$, where $H$ is a residually finite, amenable group.

(b) The surface groups

$$
T_{g}=\left\langle a_{1}, \ldots, a_{g}, b_{1}, \ldots, b_{g} \mid \prod_{i=1}^{g}\left[a_{i}, b_{i}\right]=1\right\rangle
$$

and the groups

$$
\mathrm{SL}_{2}(\mathbb{Z}[i]), \mathrm{SL}_{2}(\mathbb{Z}[\sqrt{3} i]) .
$$

Let us now show that an affirmative answer to A.4 gives a proof of 4.14.

We can assume that $[\Delta: \Gamma]=\infty$. Let $a \in A(\Delta, X, \mu)$. Consider the restriction $a \mid \Gamma \in A(\Gamma, X, \mu)$ and the co-induced action

$$
\operatorname{CInd}_{\Gamma}^{\Delta}(a \mid \Gamma) \in A\left(\Delta, X^{\Gamma / \Delta}, \mu^{\Gamma / \Delta}\right) .
$$

Let $a_{i} \in A(\Gamma, X, \mu)$ be finite actions such that $a_{i} \rightarrow a \mid \Gamma$ weakly. Let $F_{i}$ be a finite group, $b_{i} \in A\left(F_{i}, X, \mu\right)$ and $\pi_{i}: \Gamma \rightarrow F_{i}$ a surjective homomorphism such that $\gamma^{a_{i}}=\pi_{i}(\gamma)^{b_{i}}$. Let $N_{i}=\operatorname{ker}\left(\pi_{i}\right)$, so that $N_{i} \triangleleft \Gamma, F_{i}=\Gamma / N_{i}$ and $\left[\Gamma: N_{i}\right]<\infty$. By i), there is $M_{i} \triangleleft \Delta$ such that $M_{i} \subseteq N_{i}$ and $\left[\Gamma: M_{i}\right]<\infty$. It follows (by replacing $N_{i}$ by $M_{i}$ if necessary) that we can assume that $N_{i} \triangleleft \Delta$.

Lemma 4.15 Let $F$ be a finite group, $b \in A(F, X, \mu)$ and let $s_{F, X}$ be the shift action of $F$ on $X^{F}$. Then $b \prec s_{F, X}$.

Proof. Consider $F \times \mathbb{Z}$ and the action $b^{\prime} \in A(F \times \mathbb{Z}, X, \mu)$ given by $(\gamma, g)^{b^{\prime}}=\gamma^{b}$, where $(\gamma, g) \in F \times \mathbb{Z}$. Since $F \times \mathbb{Z}$ is infinite, amenable, we have that if $s_{F \times \mathbb{Z}}$ is the shift action of $F \times \mathbb{Z}$ on $2^{F \times \mathbb{Z}}$, then $b^{\prime} \prec s_{F \times \mathbb{Z}}$ (see Kechris [Ke09], 13.2). Thus $b=b^{\prime}\left|F \prec s_{F \times \mathbb{Z}}\right| F$. But clearly $s_{F \times \mathbb{Z}} \mid F$ is isomorphic to the shift action of $F$ on $\left(2^{\mathbb{Z}}\right)^{F}$ which is isomorphic to the shift action of $F$ on $X^{F}$ (since $(X, \mu)$ is non-atomic) and we are done.

It follows that $b_{i} \prec s_{F_{i}, X}$ and thus $a_{i} \prec s_{\Gamma, \Gamma / N_{i}, X}$, where $s_{\Gamma, \Gamma / N_{i}, X}$ is the (generalized) shift action of $\Gamma$ on $X^{\Gamma / N_{i}}$, defined by $\delta \cdot p\left(g N_{i}\right)=g\left(\delta^{-1} g N_{i}\right)$.

$\operatorname{Now}_{\operatorname{CInd}}^{\Delta}\left(a_{i}\right) \rightarrow \operatorname{CInd}_{\Gamma}^{\Delta}(a \mid \Gamma)$ weakly, and $\operatorname{CInd}_{\Gamma}^{\Delta}\left(a_{i}\right) \prec \operatorname{CInd}_{\Gamma}^{\Delta}\left(s_{\Gamma, \Gamma / N_{i}, X}\right)$ (see the Appendix).

By Proposition A.2 in the Appendix, $\operatorname{CInd}_{\Gamma}^{\Delta}\left(s_{\Gamma, \Gamma / N_{i}, X}\right) \cong s_{\Delta, \Delta / N_{i}, X}$, so $\operatorname{CInd}_{\Gamma}^{\Delta}\left(a_{i}\right) \prec s_{\Delta, \Delta / N_{i}, X}$. But $\Delta / N_{i}$ is infinite, amenable, and residually finite, by (ii), so $s_{\Delta / N_{i}, X}$ is a weak limit of finite actions of $\Delta / N_{i}$ and thus $s_{\Delta, \Delta / N_{i}, X}$ 
is a weak limit of finite actions of $\Delta$. It follows that each $\operatorname{CInd}_{\Gamma}^{\Delta}\left(a_{i}\right)$ is a limit of finite actions of $\Delta$ and thus so is $\operatorname{CInd}_{\Gamma}^{\Delta}(a \mid \Gamma)$. If A.4 in the Appendix has a positive answer, we have that $a \prec \operatorname{CInd}_{\Gamma}^{\Delta}(a \mid \Gamma)$. It follows that $a$ is also a weak limit of finite actions of $\Delta$ and the proof is complete.

\section{Maximality of a generalized shift action of $\mathbb{F}_{n}$}

We have seen in 3.1 that the profinite action $p_{\mathbb{F}_{n}}(1 \leq n \leq \infty)$ is maximum in the order $\prec$ of weak containment of actions of $\mathbb{F}_{n}$. We have also mentioned the result of Abert-Weiss [AW] that, for any infinite $\Gamma$, the shift action $s_{\Gamma}$ of $\Gamma$ on $2^{\Gamma}$ is minimum in the order $\prec$ among free actions. More generally, one can consider an action of $\Gamma$ on a countable set $I$ and the induced generalized shift action $s_{\Gamma, I}$ of $\Gamma$ on $2^{I}$ given by $\gamma \cdot p(i)=p\left(\gamma^{-1} \cdot i\right)$. Of special interest are the generalized shifts corresponding to transitive actions of $\Gamma$ on $I$. Equivalently these are the generalized shifts induced by the canonical action of $\Gamma$ on $I=$ $\Gamma / H$ for some $H \leq \Gamma$. We denote this by $s_{\Gamma, \Gamma / H}$. In the case of an arbitrary action of $\Gamma$ on $I$ we can decompose $I=\bigsqcup_{n} I_{n}$, into the $\Gamma$-orbits and then clearly the $\Gamma$-shift on $2^{I}=2^{\sqcup_{n} I_{n}}$ is (isomorphic to) the product of the $\Gamma$-shifts on each $2^{I_{n}}$. Thus the generalized shifts are just the products of generalized shifts of the form $s_{\Gamma, \Gamma / H}$.

Theorem 5.1 Let $\Gamma=\mathbb{F}_{n}(1 \leq n \leq \infty)$. Then there is $H \leq \Gamma$ with $[\Gamma: H]=\infty$ such that the generalized shift $s_{\Gamma, \Gamma / H}$ of $\Gamma$ on $2^{\Gamma / H}$ is maximum in the order $\prec$ of weak containment of actions of $\Gamma$.

Proof. Let $\Gamma=\mathbb{F}_{n}=\left\langle\gamma_{1}, \gamma_{2}, \ldots\right\rangle$ be free generators. We will consider the space $A(\Gamma, X, \mu)$ of measure preserving action of $\Gamma$ on $(X, \mu)$ with the weak topology. This can be identified with the product space $\operatorname{Aut}(X, \mu)^{n}$ (where $n$ denotes $\{1, \ldots, n\}$, if $n<\infty$, and $\mathbb{N} \backslash\{0\}$, if $n=\infty)$, with $\operatorname{Aut}(X, \mu)$ again equipped with the weak topology, by identifying $a \in A(\Gamma, X, \mu)$ with $\left(\gamma_{i}^{a}\right)_{i} \in \operatorname{Aut}(X, \mu)^{n}$.

We say that $a \in A(\Gamma, X, \mu)$ factors through a group $\Delta$ if there is a surjective homomorphism $\rho: \Gamma \rightarrow \Delta$ and $b \in A(\Delta, X, \mu)$ such that $\forall \gamma \in \Gamma\left(\gamma^{a}=\right.$ $\left.\rho(\gamma)^{b}\right)$. We will say that $a \in A(\Gamma, X, \mu)$ factors regularly through $\Delta$ if the above holds but additionally $\rho\left(\gamma_{i}\right)$ has infinite order in $\Delta, \forall i$. In that case we call $\rho$ regular. 
Lemma 5.2 The actions of $\Gamma$ on $(X, \mu)$ that factor regularly through an infinite amenable group are weakly dense in $A(\Gamma, X, \mu)$.

Proof. By $\S 4$ the group $\Gamma$ has property MD, thus the finite actions are weakly dense in $A(\Gamma, X, \mu)$. Consider such an action $a \in A(\Gamma, X, \mu)$, let $\sigma: \Gamma \rightarrow F$ be a surjective homomorphism, where $F$ is finite, and let $c \in A(F, X, \mu)$ be such that $\gamma^{a}=\sigma(\gamma)^{c}, \forall \gamma \in \Gamma$. Let $\Delta_{1}=F \times \mathbb{Z}$ and let $\pi: \Delta_{1} \rightarrow F$ be the projection to the first coordinate. Let $g \neq 0$ be an element of $\mathbb{Z}$. Let $\tau: \Gamma \rightarrow \Delta_{1}$ be the homomorphism defined by $\tau\left(\gamma_{i}\right)=\left(\sigma\left(\gamma_{i}\right), g\right)$, so that $\tau\left(\gamma_{i}\right)$ has infinite order. Let $\tau(\Gamma)=\Delta \subseteq \Delta_{1}$. Thus $\Delta$ is an infinite amenable group, and if $b \in A(\Delta, X, \mu)$ is defined by $\delta^{b}=\pi(\delta)^{c}$, then, as $\sigma=\pi \circ \tau$, we have $\gamma^{a}=\sigma(\gamma)^{c}=(\pi(\tau(\gamma)))^{c}=\tau(\gamma)^{b}$, i.e., a factors regularly through $\Delta$.

Suppose now $\rho: \Gamma \rightarrow \Delta$ is a regular surjective homomorphism, where $\Delta$ is infinite amenable, $b \in A(\Delta, X, \mu)$ and $a \in A(\Gamma, X, \mu)$ is given by $\gamma^{a}=\rho(\gamma)^{b}$. Since $\Delta$ is infinite amenable, $b \prec s_{\Delta}$, where $s_{\Delta}$ is the shift action of $\Delta$ on $2^{\Delta}$ (see Kechris [Ke09], 13.2). Composing with $\rho$, we see that $a \prec s_{\Gamma, \Gamma / N}$, where $N=\operatorname{ker}(\rho)$.

To simplify notation, we will work from now on with $\Gamma=\mathbb{F}_{2}=\left\langle\gamma_{1}, \gamma_{2}\right\rangle$. The general case requirers only trivial modifications.

Fix a countable open basis $\left\{U_{n}\right\}$ for $A(\Gamma, X, \mu)$, so that every $U_{n}$ has the following form:

$$
\begin{aligned}
U_{n}=\left\{a \in A(\Gamma, X, \mu): \forall i \leq 2 \forall j, k \leq m_{n} \mid \mu\left(\gamma_{i}^{a}\left(P_{j}^{(n)}\right)\right.\right. & \left.\cap P_{k}^{(n)}\right)- \\
\mu\left(\gamma_{i}^{a_{n}}\left(P_{j}^{(n)}\right)\right. & \left.\cap P_{k}^{(n)}\right) \mid<\epsilon_{n},
\end{aligned}
$$

where $a_{n} \in A(\Gamma, X, \mu), \mathcal{P}_{n}=\left\{P_{1}^{(n)}, \ldots, P_{m_{n}}^{(n)}\right\}$ is a Borel partition of $X$ and $\epsilon_{n}>0$ (see Kechris [Ke09], Section 1, (B)).

From 5.2, and the paragraph following it, it follows that for each $n$, there is an infinite set $I_{n}$, a transitive action $\tau_{n}$ of $\Gamma$ on $I_{n}$ such that no $\gamma_{i}^{\tau_{n}}(i \leq 2)$ has an invariant finite set (this is where regularity is used) and is such that an isomorphic copy $\bar{a}_{n}$ of $s_{\Gamma, I_{n}}$ is in $U_{n}$, say via the isomorphism $\varphi_{n}:\left(2^{I_{n}}, \nu_{n}\right) \rightarrow$ $(X, \mu)$, where $\nu_{n}$ is the usual product measure on $2^{I_{n}}$. Let $\varphi_{n}^{-1}\left(\mathcal{P}_{n}\right)=\mathcal{R}_{n}=$ $\left\{R_{1}^{(n)}, \ldots R_{m_{n}}^{(n)}\right\}$ be the partition of $2^{I_{n}}$ in which $R_{j}^{(n)}=\varphi_{n}^{-1}\left(P_{j}^{(n)}\right)$, so that $\forall i \leq$ $2 \forall j, k \leq m_{n}, \nu_{n}\left(R_{j}^{(n)}\right)=\mu\left(P_{j}^{(n)}\right)$ and $\mid \nu_{n}\left(\gamma_{i}^{s_{\Gamma, I_{n}}}\left(R_{j}^{(n)}\right) \cap R_{k}^{(n)}\right)-\mu\left(\gamma_{i}^{a_{n}}\left(P_{j}^{(n)}\right) \cap\right.$ $\left.P_{k}^{(n)}\right) \mid<\rho_{n}<\epsilon_{n}$.

A basic nbhd of $2^{I_{n}}$ is a set of the form $\left\{p \in 2^{I_{n}}: p \mid F=u\right\}$, where $u \in 2^{F}$, $F$ finite. A finite union of such basic nbhds, with the same $F$, will be called 
a clopen set supported by $F$. Since the clopen sets with finite support are dense in the measure algebra of $2^{I_{n}}$, it follows that for every $\delta>0$, there is a finite non-empty set $F_{n, \delta}$ and a partition of $2^{I_{n}}$ into clopen sets supported by $F_{n, \delta}, \mathcal{S}_{n, \delta}=\left\{S_{1, \delta}^{(n)} \ldots, S_{m_{n}, \delta}^{(n)}\right\}$, such that $\forall j \leq m_{n}\left(\nu_{n}\left(R_{j}^{(n)} \Delta S_{j, \delta}^{(n)}\right)<\delta\right)$, thus $\forall i \leq 2 \forall j, k \leq m_{n}\left(\left|\nu_{n}\left(\gamma_{i}^{s_{\Gamma}, I_{n}}\left(S_{j, \delta}^{(n)}\right) \cap S_{k, \delta}^{(n)}\right)-\nu_{n}\left(\gamma_{i}^{s_{\Gamma}, I_{n}}\left(R_{j}^{(n)}\right) \cap R_{k}^{(n)}\right)\right|<2 \delta\right)$.

Let $\overline{\mathcal{P}}_{n, \delta}=\varphi_{n}\left(\mathcal{S}_{n, \delta}\right)=\left\{\bar{P}_{1, \delta}^{(n)}, \ldots, \bar{P}_{m_{n}, \delta}^{(n)}\right\}$ be the partition of $X$ in which $\bar{P}_{j, \delta}^{(n)}=\varphi_{n}\left(S_{j, \delta}^{(n)}\right)$. Then $\mu\left(\bar{P}_{j, \delta}^{n} \Delta P_{j}^{n}\right)<\delta, \forall j \leq m_{n}$, so $\forall i \leq 2 \forall j, k \leq m_{n}$,

$$
\left|\mu\left(\gamma_{i}^{\bar{a}_{n}}\left(\bar{P}_{j, \delta}^{(n)}\right) \cap \bar{P}_{k, \delta}^{(n)}\right)-\mu\left(\gamma_{i}^{a_{n}}\left(\bar{P}_{j, \delta}^{(n)}\right) \cap \bar{P}_{k, \delta}^{(n)}\right)\right|<\rho_{n}+4 \delta .
$$

Fix $\delta=\delta_{n}$ and $\epsilon_{n}^{\prime}$, so that $\rho_{n}+4 \delta_{n}<\epsilon_{n}^{\prime}<\epsilon_{n}^{\prime}+4 \delta_{n}<\epsilon_{n}$. Let $U_{n}^{\prime}$ be the set of all $\left\{a \in A(\Gamma, X, \mu)\right.$ such that $\forall i \leq 2 \forall j, k \leq m_{n}$

$$
\mid \mu\left(\gamma_{i}^{a}\left(\bar{P}_{j, \delta_{n}}^{(n)} \cap \bar{P}_{k, \delta_{n}}^{(n)}\right)-\mu\left(\gamma_{i}^{a_{n}}\left(\bar{P}_{j, \delta_{n}}^{(n)}\right) \cap \bar{P}_{k, \delta_{n}}^{(n)}\right) \mid<\epsilon_{n}^{\prime}\right\} .
$$

Then $\bar{a}_{n} \in U_{n}^{\prime}$ and $U_{n}^{\prime} \subseteq U_{n}$. Put $\bar{P}_{j}^{(n)}=\bar{P}_{j, \delta_{n}}^{(n)}, \overline{\mathcal{P}}_{n}=\mathcal{P}_{n, \delta_{n}}, F_{n}=F_{n, \delta_{n}}, \mathcal{S}_{n}=$ $\mathcal{S}_{n, \delta_{n}}$. Thus $\varphi_{n}: 2^{I_{n}} \rightarrow X$ sends $\mathcal{S}_{n}$ to $\overline{\mathcal{P}}_{n}, \mathcal{S}_{n}$ is supported by $F_{n}$ and $\varphi_{n}$ sends $s_{\Gamma, I_{n}}$ to $\bar{a}_{n}$, where

$$
\left|\mu\left(\gamma_{i}^{\bar{a}_{n}}\left(\bar{P}_{j}^{(n)}\right) \cap \bar{P}_{k}^{(n)}\right)-\mu\left(\gamma_{i}^{a_{n}}\left(\bar{P}_{j}^{(n)}\right) \cap \bar{P}_{k}^{(n)}\right)\right|<\epsilon_{n}^{\prime},
$$

$\forall i \leq 2 \forall j, k \leq m_{n}$. Also $\mu\left(\bar{P}_{j}^{(n)}\right)=\nu_{n}\left(S_{j}^{(n)}\right)$.

Now consider the product action $s=\prod_{n} s_{\Gamma, I_{n}}$, which is the action of $\Gamma$ on $2^{I}, I=\bigsqcup_{n} I_{n}$, induced by the action $\tau$ of $\Gamma$ on $I$ given by $\tau=\bigsqcup_{n} \tau_{n}$. For each $n$, let $p_{n}: 2^{I} \rightarrow 2^{I_{n}}$ be the projection function $p_{n}(f)=f \mid I_{n}$. Let $\overline{\mathcal{S}}_{n}=p_{n}^{-1}\left(\mathcal{S}_{n}\right)=\left\{p_{n}^{-1}\left(S_{1}^{(n)}\right), \ldots, p_{n}^{-1}\left(S_{m_{n}}^{(n)}\right)\right\}$. This is a clopen partition of $2^{I}$ supported by the finite set $F_{n} \subseteq I_{n} \subseteq I$. If $\nu=\prod_{n} \nu_{n}$ is the product measure on $2^{I}$, clearly $\forall i \leq 2 \forall j, k \leq m_{n}\left(\nu\left(\bar{S}_{j}^{(n)}\right)=\nu_{n}\left(S_{j}^{(n)}\right)\right)$ and $\nu_{n}\left(\gamma_{i}^{s_{\Gamma}, I_{n}}\left(S_{j}^{(n)}\right) \cap\right.$ $\left.S_{k}^{(n)}\right)=\nu\left(\gamma_{i}^{s}\left(\bar{S}_{j}^{(n)}\right) \cap \bar{S}_{k}^{(n)}\right)$.

Now each $\bar{S}_{j}^{(n)}$ is a finite union of basic nbhds of the form $N_{u}=\{f \in$ $\left.2^{I}: f \mid F_{n}=u\right\}$, where $u \in 2^{F_{n}}$. Since $\gamma_{i}^{s}\left(N_{u}\right)=N_{v}$, where $v \in 2^{\gamma_{i}^{\tau_{n}}\left(F_{n}\right)}$ is defined by $v(a)=u\left(\left(\gamma_{i}^{-1}\right)^{\tau_{n}}(a)\right)$, it follows that $\gamma_{i}^{s}\left(N_{u}\right)$, for each such $u$, and thus $\gamma_{i}^{s}\left(\bar{S}_{j}^{(n)}\right), \forall j \leq m_{n}$, depends only on $\gamma_{i}^{\tau}\left|F_{n}=\gamma_{i}^{\tau_{n}}\right| F_{n}$.

So fix any action $\tau^{*}$ of $\Gamma$ on $I$ such that $\gamma_{i}^{\tau^{*}}\left|F_{n}=\gamma_{i}^{\tau}\right| F_{n}=\gamma_{i}^{\tau_{n}} \mid F_{n}, \forall n \forall i \leq$ 2 , and let $s^{*}$ be the corresponding shift action on $2^{I}$. Then $\gamma_{i}^{s}\left(\bar{S}_{j}^{(n)}\right)=$ $\gamma_{i}^{s^{*}}\left(\bar{S}_{j}^{(n)}\right), \forall n \forall i \leq 2 \forall j \leq m_{n}$. 
Lemma 5.3 For each $n$ there is an isomorphic copy of $s^{*}$ in $U_{n}^{\prime}$, thus $s^{*}$ is maximum in $\prec$.

Proof. Fix $n$. We will find an isomorphic copy of $s^{*}$ in $U_{n}^{\prime}$. Since $\mu\left(\bar{P}_{j}^{(n)}\right)=\nu_{n}\left(S_{j}^{(n)}\right)=\nu\left(\bar{S}_{j}^{(n)}\right)$, let $\psi_{n}:\left(2^{I}, \nu\right) \rightarrow(X, \mu)$ be an isomorphism such that $\psi_{n}\left(\bar{S}_{j}^{(n)}\right)=\bar{P}_{j}^{(n)}, \forall j \leq m_{n}$. Let $a_{n}^{*} \in A(\Gamma, X, \mu)$ be the isomorphic copy of $s^{*}$ induced by $\psi_{n}$. We will check that $a_{n}^{*} \in U_{n}^{\prime}$. Indeed, $\mu\left(\gamma_{i}^{a_{n}^{*}}\left(\bar{P}_{j}^{(n)}\right) \cap\right.$ $\left.\bar{P}_{k}^{(n)}\right)=\nu\left(\gamma_{i}^{s^{*}}\left(\bar{S}_{j}^{(n)}\right) \cap \bar{S}_{k}^{(n)}\right)=\nu\left(\gamma_{i}^{s}\left(\bar{S}_{j}^{(n)}\right) \cap \bar{S}_{k}^{(n)}\right)=\nu_{n}\left(\gamma_{i}^{s_{\Gamma}, I_{n}}\left(S_{j}^{(n)}\right) \cap S_{k}^{(n)}\right)=$ $\mu\left(\gamma_{i}^{\bar{a}_{n}}\left(\bar{P}_{j}^{(n)}\right) \cap \bar{P}_{k}^{(n)}\right)$, and so $\left|\mu\left(\gamma_{i}^{a_{n}^{*}}\left(\bar{P}_{j}^{(n)}\right) \cap \bar{P}_{k}^{(n)}\right)-\mu\left(\gamma_{i}^{a_{n}}\left(\bar{P}_{j}^{(n)}\right) \cap \bar{P}_{k}^{(n)}\right)\right|<\epsilon_{n}^{\prime}$, i.e., $a_{n}^{*} \in U_{n}^{\prime}$.

To complete the proof, it is enough to show that such a $\tau^{*}$ can be chosen so that $\tau^{*}$ acts transitively on $I$. Let us recall that $I=\bigsqcup_{n} I_{n}, I_{n}$ infinite, $\emptyset \neq F_{n} \subseteq I_{n}$ is finite, $\tau=\bigsqcup_{n} \tau_{n}, \gamma_{i}^{\tau_{n}}$ does not have any finite (non- $\emptyset$ ) invariant sets $\left(\subseteq I_{n}\right)$ and we want to find $\tau^{*}$, a transitive action of $\Gamma$ on $I$, such that $\forall i \leq 2 \forall n\left(\gamma_{i}^{\tau^{*}}\left|F_{n}=\gamma_{i}^{\tau}\right| F_{n}\right)$. First we take $\gamma_{1}^{\tau^{*}}=\gamma_{1}^{\tau}$. It is then enough to find $\gamma_{2}^{\tau^{*}}$, a transitive permutation of $I$, that satisfies $\gamma_{2}^{\tau^{*}}\left|F_{n}=\gamma_{2}^{\tau}\right| F_{n}$, for each $n$. This is possible by the following lemma.

Lemma 5.4 For each $n \geq 1$, let $I_{n}$ be an infinite (countable) set, $\emptyset \neq F_{n} \subseteq$ $I_{n}$ a finite subset and $S_{n}$ a permutation of $I_{n}$ that has no finite (non- $\emptyset$ ) invariant sets. Then there is a transitive permutation $S$ of I such that $S \mid F_{n}=$ $S_{n} \mid F_{n}, \forall n$.

Proof. Because $S_{n}$ has no finite non- $\emptyset$ invariant sets, i.e., no cycles, there is a partition $P_{1}^{(n)}, \ldots, P_{k_{n}}^{(n)}$ of $F_{n} \cup S_{n}\left(F_{n}\right)$, such that each $P_{i}^{(n)}$ has the form $\left\{x_{i}^{(n)}, S_{n}\left(x_{i}^{(n)}\right), \ldots, S_{n}^{\ell_{n}}\left(x_{i}^{(n)}\right)=y_{i}^{(n)}\right\}$, for some $\ell_{n}$, where all the $S_{n}^{j}\left(x_{i}^{(n)}\right)$ are distinct and $x_{i}^{(n)} \notin S_{n}\left(F_{n}\right), y_{i}^{(n)} \notin F_{n}$. Call $x_{i}^{(n)}, y_{i}^{(n)}$ the first, resp., last, elements of $P_{i}^{(n)}$. Clearly $S_{n}\left|F_{n}=\bigcup_{i \leq k_{n}} S_{n}\right|\left(P_{i}^{(n)} \backslash\left\{y_{i}^{(n)}\right\}\right)$. Put $P=I \backslash \bigcup_{n, i \leq k_{n}} P_{i}^{(n)}$ and enumerate $P=\left\{p_{0}, p_{1}, \ldots\right\}$. Then let $S$ be defined as follows: $S\left(p_{i+1}\right)=p_{i}$ for $i \geq 0, S\left|\left(P_{i}^{(n)} \backslash\left\{y_{i}^{(n)}\right\}\right)=S_{n}\right|\left(P_{i}^{(n)} \backslash\left\{y_{i}^{(n)}\right\}\right)$ (thus $\left.S\left|F_{n}=S_{n}\right| F_{n}\right)$, and finally $S\left(p_{0}\right)=x_{1}^{(1)}, S\left(y_{1}^{(1)}\right)=x_{2}^{(1)}, S\left(y_{2}^{(1)}\right)=$ $x_{3}^{(1)}, \ldots, S\left(y_{k_{1}}^{(1)}\right)=x_{1}^{(2)}, S\left(y_{1}^{(2)}\right)=x_{2}^{(2)}, \ldots$

By a similar argument, using direct sums of representations instead of product actions, one can show that for $\Gamma=\mathbb{F}_{n}(1 \leq n \leq \infty)$, there is $H \leq \Gamma$ with $[\Gamma: H]=\infty$ such that the quasi-regular representation $\lambda_{\Gamma / H}$ on $\ell^{2}(\Gamma / H)$, induced by the canonical action of $\Gamma$ on $\Gamma / H$, is maximum in 
the order $\prec$ of weak containment of unitary representations of $\Gamma$. Monod (private communication) asked whether, for $n \geq 2$, one can also find such an $H$ such that moreover $\lambda_{\Gamma / H}$ is irreducible. (The existence of irreducible representations of $\mathbb{F}_{n}$, for $n \geq 2$, that are maximum in the order of weak containment was first proved by Yoshizawa [Y]. For another proof, see Kechris [Ke09], Appendix H, (C)). One can easily modify the preceding argument to show that this is indeed the case. (I would like to thank T. Tsankov for a helpful discussion on this matter.)

Theorem 5.5 Let $\Gamma=\mathbb{F}_{n}(2 \leq n \leq \infty)$. Then there is $H \leq \Gamma$ with $[\Gamma: H]=\infty$ such that the quasi-regular representation $\lambda_{\Gamma / H}$ is maximum in the order $\prec$ of weak containment of unitary representations of $\Gamma$ and is moreover irreducible..

Proof. We again take $\Gamma=\mathbb{F}_{2}=\left\langle\gamma_{1}, \gamma_{2}\right\rangle$ for notational simplicity. As in the proof of 5.1, we can find a sequence of infinite sets $\left\{I_{n}\right\}$, a transitive action $\tau_{n}$ of $\Gamma$ on $I_{n}$ such that no $\gamma_{i}^{\tau_{n}}(i \leq 2)$ has an invariant finite set, and a sequence $\left\{F_{n}\right\}$ of non-empty finite sets with $F_{n} \subseteq I_{n}, \forall n$, and such that if $\tau^{*}$ is any transitive action of $\Gamma$ on $I=\bigsqcup_{n} I_{n}$ with $\forall i \leq 2 \forall n\left(\gamma_{i}^{\tau^{*}} \mid F_{n}=\right.$ $\left.\gamma_{i}^{\tau_{n}} \mid F_{n}\right)$, then the representation $\lambda_{\Gamma / H}$, where $H$ is the stabilizer in $\tau^{*}$ of some (equivalently any) point of $I$, is maximum in the order of weak containment of representations of $\mathbb{F}_{2}$. By Mackey's Irreducibility Criterion (see, e.g., Bekkade la Harpe [BdlH], Example 10), in order to make $\lambda_{\Gamma / H}$ irreducible, it is enough to have that the action of $H$ on $\Gamma / H$ has infinite orbits except on $H$ itself. In terms of the action $\tau^{*}$, it is enough to find a point $i_{0} \in I$ whose stabilizer $H$ has the property that the orbits of $\tau^{*} \mid H$ are infinite on $I \backslash\left\{i_{0}\right\}$.

By a simple modification of the proof of 5.4 (by taking $S_{n}=\gamma_{2}^{\tau_{n}} \mid I_{n}$ ), we see that we can find finite sets $\tilde{P}_{n}\left(=\bigcup_{i \leq k_{n}} P_{i}^{(n)}\right.$, in the notation of that proof), with $\tilde{P}_{n} \subseteq I_{n}$, and for each $i_{0} \in P=I \backslash \bigcup_{n} \tilde{P}_{n}$, we can find $\gamma_{2}^{\tau^{*}}$ such that $\gamma_{2}^{\tau^{*}}\left|F_{n}=\gamma_{2}^{\tau}\right| F_{n}$ and $\gamma_{2}^{\tau^{*}}$ fixes $i_{0}$ and acts transitively on the rest of $I$. Then the stabilizer $H$ of $i_{0}$ contains $\gamma_{2}$ and thus (no matter how we define $\left.\gamma_{1}^{\tau^{*}}\right) \tau^{*} \mid H$ has a single orbit off $i_{0}$. We only need now to define $\gamma_{1}^{\tau^{*}}$ to make sure that the action $\tau^{*}$ is transitive and of course also have $\gamma_{1}^{\tau^{*}}\left|F_{n}=\gamma_{i}^{\tau}\right| F_{n}$, for every n. But this is clear from 5.4 again.

Recall that the action of a group $\Gamma$ on $\Gamma / H$ is amenable if $1_{\Gamma} \prec \lambda_{\Gamma / H}$. If we now take $\Gamma=\mathbb{F}_{n}$ and $H$ as in 5.5, then $\pi \prec \lambda_{\Gamma / H}$ for every unitary representation $\pi$, so, in particular, $1_{\Gamma} \prec \lambda_{\Gamma / H}$, i.e., the action of $\Gamma$ on $I$ is amenable. Since we also have that $\lambda_{\Gamma} \prec \lambda_{\Gamma / H}$, it is easy to check that no 
$\gamma \neq 1$ fixes every element of $\Gamma / H$, i.e., the action of $\Gamma$ on $\Gamma / H$ is faithful. Thus we see that the action of $\Gamma$ on $I=\Gamma / H$, where $H$ comes from 5.5, gives another example of a faithful, transitive, amenable action of $\mathbb{F}_{n}$ on a countable set $I$, a result first proved by van Douwen [vD]. Other such examples have been found in Glasner-Monod [GM] and Grigorchuk-Nekrashevych [GN]. However, the example coming from 5.5 has the stronger property that $\forall \pi\left(\pi \prec \lambda_{\Gamma / H}\right)$ instead of just $1_{\Gamma} \prec \lambda_{\Gamma / H}$ and is also irreducible.

We finally note that in 5.1, the action $s_{\Gamma, \Gamma / H}$ is weakly mixing (see, e.g., Kechris-Tsankov [KT], 2.1). One can also make it free by using 2.4, (ii) in Kechris-Tsankov $[\mathrm{KT}]$ (or else work with $s_{\Gamma / \Gamma / H, X}$, for $(X, \mu)$ non-atomic, in which case $s_{\Gamma, \Gamma / H, X}$ is automatically free, as the action of $\Gamma$ on $\Gamma / H$ is faithful (see $[\mathrm{KT}], 2.4$, (iii))).

\section{Miscellanea}

We will consider here some additional density properties in the space of actions $A\left(\mathbb{F}_{n}, X, \mu\right)$ of the free group $\mathbb{F}_{n}$ and some of its subspaces.

Given a countable, measure preserving equivalence relation $E$ on $(X, \mu)$, we denote by $[E]$ its full group,

$$
[E]=\{T \in \operatorname{Aut}(X, \mu): T(x) E x, \mu \text {-a.e. }(x)\} .
$$

For any group $\Gamma$, we let $A(\Gamma,[E])$ be the space of actions of $\Gamma$ "contained" in $[E]$ :

$$
A(\Gamma,[E])=\left\{a \in A(\Gamma, X, \mu): \forall \gamma \in \Gamma\left(\gamma^{a} \in[E]\right)\right\} .
$$

Since $[E]$ is a separable subgroup in the uniform topology of $\operatorname{Aut}(X, \mu)$, it follows that $A(\Gamma,[E])$ is a separable (thus Polish) space in the uniform topology of $A(\Gamma, X, \mu)$. When $\Gamma=\mathbb{F}_{n}(1 \leq n \leq \infty)$, with free generators $\gamma_{1}, \gamma_{2}, \ldots$, we also let $\operatorname{AP}\left(\mathbb{F}_{n},[E]\right)$ be the uniformly closed subspace of $A\left(\mathbb{F}_{n},[E]\right)$ consisting of all $a \in A\left(\mathbb{F}_{n},[E]\right)$ for which $\gamma_{1}^{a}$ is aperiodic. Denoting by APER the (uniformly closed) set of aperiodic elements of $\operatorname{Aut}(X, \mu)$, we can clearly identify $\operatorname{AP}\left(\mathbb{F}_{n},[E]\right)$ with $(\operatorname{APER} \cap[E]) \times[E]^{n-1}$ (with the product of the uniform topology).

(A) We will first consider any equivalence relation $E$ on $(X, \mu)$ of cost $C(E)>1$. We note that, by the argument in Ioana-Peterson-Popa [IPP], Appendix, and Gaboriau $[\mathrm{G}]$, it follows that for any equivalence relation $F$ there is an equivalence relation $F \subseteq E$ with $C(E)>1$. 
Proposition 6.1 Let $E$ be a countable, measure preserving, ergodic equivalence relation on $(X, \mu)$ with $C(E)>1$. Then for each $2 \leq n \leq \infty,\{a \in$ $\left.\operatorname{AP}\left(\mathbb{F}_{n},[E]\right): C(a)>1\right\}$ is dense and non-meager in the uniform topology of $\operatorname{AP}\left(\mathbb{F}_{n},[E]\right)$.

Proof. We will consider the case $n=2$, the case of arbitrary $n$ being similar.

(1) First we will prove density.

Claim. There is $a \in \mathrm{AP}\left(\mathbb{F}_{2},[E]\right)$ such that $E_{a}$ is maximal (under a.e. inclusion) with the property that $E_{a}$ is ergodic and $C\left(E_{a}\right)=1$.

Proof. Assume not. Let $a_{0} \in \operatorname{AP}\left(\mathbb{F}_{2},[E]\right)$ be such that $E_{a_{0}}$ is ergodic and $C\left(E_{a_{0}}\right)=1$ (such exists using, e.g., Kechris [Ke09], 3.5). Then by transfinite induction on $\alpha<\omega_{1}$ (the first uncountable ordinal), we will find $a_{\alpha} \in \operatorname{AP}\left(\mathbb{F}_{2},[E]\right)$ such that $C\left(E_{a_{\alpha}}\right)=1, \alpha<\beta \Rightarrow E_{a_{\alpha}} \varsubsetneqq E_{a_{\beta}}$, and $E_{a_{\lambda}}=$ $\bigcup_{\alpha<\lambda} E_{a_{\alpha}}$ for $\lambda$ limit. The successor case is trivial. For the limit case, let first $E_{\lambda}=\bigcup_{\alpha<\lambda} E_{a_{\alpha}}$. Then $C\left(E_{\lambda}\right)=1$ (see Gaboriau [G] or Kechris-Miller [KM], 23.5). It is thus enough to find $a_{\lambda} \in \operatorname{AP}\left(\mathbb{F}_{2},[E]\right)$ with $E_{a_{\lambda}}=E_{\lambda}$. Note that $E_{\lambda}$ is ergodic. So let $S_{\lambda} \in\left[E_{\lambda}\right]$ be ergodic. Since $C\left(E_{\lambda}\right)<3 / 2$, by the proof of Kechris-Miller $[\mathrm{KM}], 27.7$, there is $\varphi_{\lambda} \in\left[\left[E_{\lambda}\right]\right]$, such that $E_{\lambda}$ is the equivalence relation generated by $S_{\lambda}, \varphi_{\lambda}$. If $E_{\varphi_{\lambda}}$ is the equivalence relation generated by $\varphi_{\lambda}$, then since $E_{\varphi_{\lambda}}$ is a hyperfinite subrelation of $E_{\lambda}$, it is easy to find an aperiodic $T_{\lambda} \in\left[E_{\lambda}\right]$ such that $E_{\varphi_{\lambda}} \subseteq E_{T_{\lambda}}$. So if $\mathbb{F}_{2}=\left\langle\gamma_{1}, \gamma_{2}\right\rangle$ and we let $a_{\lambda}$ be defined by $\gamma_{1}^{a_{\lambda}}=S_{\lambda}, \gamma_{2}^{a_{\lambda}}=T_{\lambda}$, then clearly $a \in \operatorname{AP}\left(\mathbb{F}_{2},[E]\right), E_{a_{\lambda}}=E_{\lambda}$.

The existence of $a_{\lambda}, \lambda<\omega_{1}$, clearly violates the countable chain condition in the $\sigma$-finite measure space $(E, M)$, where $M(A)=\int \operatorname{card}\left(A_{x}\right) d \mu(x)$, with $A_{x}=\{y:(x, y) \in A\}$ for any Borel set $A \subseteq E$.

So fix $a \in \operatorname{AP}\left(\mathbb{F}_{2},[E]\right)$ as in the claim. Note that $E_{a} \varsubsetneqq E$, from which it follows that $[E] \backslash\left[E_{a}\right] \neq \emptyset$. Let $\gamma_{1}^{a}=S_{1}, \gamma_{2}^{a}=S_{2}$.

Claim. If $S_{2}^{\prime} \in[E] \backslash\left[E_{a}\right]$ and $a^{\prime}$ is defined by $\gamma_{1}^{a^{\prime}}=S_{1}, \gamma_{2}^{a^{\prime}}=S_{2}^{\prime}$, then $C\left(a^{\prime}\right)>1$.

Proof. If not, consider $E^{\prime}=E_{a} \vee E_{a^{\prime}}$. Then, since $E_{a} \cap E_{a^{\prime}}$ is aperiodic, $C\left(E^{\prime}\right)=1$ (see Gaboriau [G] or Kechris-Miller [KM], 23.4) and as in the proof of the previous claim $E^{\prime}=E_{b}$, for some $b \in \mathrm{AP}\left(\mathbb{F}_{2},[E]\right)$, and clearly $E_{a} \varsubsetneqq E_{b}$, contradicting the maximality of $a$.

We now complete the proof of density. Fix any $a_{0} \in \operatorname{AP}\left(\mathbb{F}_{2},[E]\right)$, with $\gamma_{1}^{a_{0}}=S_{1}^{0}, \gamma_{2}^{a_{0}}=S_{2}^{0}$. Fix also uniform open nbhds $U_{1}, U_{2}$ of $S_{1}^{0}, S_{2}^{0}$, resp. We 
will find $c \in \operatorname{AP}\left(\mathbb{F}_{2},[E]\right)$ with $C(c)>1$ such that $\gamma_{1}^{c} \in U_{1}, \gamma_{2}^{c} \in U_{2}$. Since the conjugates of any aperiodic $S \in[E]$ by elements of $[E]$ are uniformly dense in APER $\cap[E]$ (see Kechris [Ke09], 3.4), we can assume that $\gamma_{1}^{a}=S_{1} \in U_{1}$ (by replacing $a$ by a conjugate action within $[E]$ if necessary). Now $\left[E_{a}\right]$ is a uniformly closed proper subgroup of $[E]$, so it must have empty interior, otherwise it would be open, thus clopen, violating the connectedness of $[E]$ in the uniform topology (see Kechris [Ke09], 3.12). Thus $[E] \backslash\left[E_{a}\right]$ is uniformly dense in $[E]$ and so $U_{2} \cap\left([E] \backslash\left[E_{a}\right]\right) \neq \emptyset$. Then pick $T_{2} \in U_{2} \cap\left([E] \backslash\left[E_{a}\right]\right)$. Let $c \in \operatorname{AP}\left(\mathbb{F}_{2},[E]\right)$ be such that $\gamma_{1}^{c}=\gamma_{1}^{a_{0}}=S_{1} \in U_{1}, \gamma_{2}^{c}=T_{2} \in U_{2}$. By the previous claim $C(c)>1$ and we are done.

(2) Next we prove non-meagerness.

It will be convenient to use the following notation: For any topological space $X$ and $P \subseteq X$, we put

$$
\forall^{*} x \in X P(x) \Leftrightarrow P \text { is comeager in } X \text {. }
$$

We also let

$$
A=\mathrm{APER} \cap[E],
$$

so that $\operatorname{AP}\left(\mathbb{F}_{2},[E]\right)$ can be identified with $A \times[E]$. All these spaces are equipped with the uniform topology.

Assume that $\left\{a \in \operatorname{AP}\left(\mathbb{F}_{2},[E]\right): C(a)=1\right\}$ is comeager, towards a contradiction. Then letting for each $V_{0}, V_{1}, \cdots \in \operatorname{Aut}(X, \mu), E_{V_{0}, V_{1}, \ldots}$ be the equivalence relation generated by $V_{0}, V_{1}, \ldots$, we have

$$
\forall^{*}(S, T) \in A \times[E]\left(C\left(E_{S, T}\right)=1\right),
$$

so by the Kuratowski-Ulam Theorem

$$
\forall^{*} S \in A \forall^{*} T \in[E]\left(C\left(E_{S, T}\right)=1\right) .
$$

Claim. For any n,

$$
\forall^{*} S \in A \forall^{*} T_{0} \in[E] \cdots \forall^{*} T_{n} \in[E]\left(C\left(E_{S, T_{0}, T_{1}, \ldots, T_{n}}\right)=1\right) .
$$

Proof. By induction on $n$. This is clear for $n=0$. Assume it is true for $n$. Then using this and the $n=0$ case, we have

$$
\begin{gathered}
\forall^{*} S \in A \forall^{*} T_{0} \in[E] \cdots \forall^{*} T_{n} \in[E] \forall^{*} T_{n+1} \in[E] \\
\left(C\left(E_{S . T_{0}, \ldots, T_{n}}\right)=1 \wedge C\left(E_{S, T_{n+1}}\right)=1\right) .
\end{gathered}
$$


For $S, T_{0}, \ldots, T_{n+1}$ as above,

$$
E_{S . T_{0}, \ldots, T_{n}} \cap E_{S, T_{n+1}} \supseteq E_{S}
$$

and

$$
E_{S, T_{0}, \ldots, T_{n}, T_{n+1}}=E_{S, T_{0}, \ldots, T_{n}} \vee E_{S, T_{n+1}},
$$

while $E_{S}$ is aperiodic. Thus it follows as before that

$$
C\left(E_{S, T_{0}, \ldots, T_{n+1}}\right)=1,
$$

i.e.,

$$
\forall^{*} S \in A \forall^{*} T_{0} \in[E] \cdots \forall^{*} T_{n+1} \in[E]\left(C\left(E_{S, T_{0}, \ldots, T_{n+1}}\right)=1\right)
$$

Using this claim, we then have

$$
\forall^{*} S \in A \forall^{*}\left(T_{0}, T_{1}, \ldots\right) \in[E]^{\mathbb{N}}\left(C\left(E_{S, T_{0}, T_{1}, \ldots}\right)=1\right),
$$

since $E_{S, T_{0}, T_{1}, \ldots}=\bigcup_{n} E_{S, T_{0}, T_{1}, \ldots, T_{n}}$ and $\left\{E_{S, T_{0}, T_{1}, \ldots, T_{n}}\right\}$ is an increasing sequence of cost 1 equivalence relations for comeager many $\left(S,\left(T_{0}, T_{1}, \ldots\right)\right) \in A \times[E]^{\mathbb{N}}$.

On the other hand, we have

$$
\forall^{*} S \in A \forall^{*}\left(T_{0}, T_{1}, \ldots\right) \in[E]^{\mathbb{N}}\left(E_{S, T_{0}, T_{1}, \ldots}=E\right)
$$

which is a contradiction, since $C(E)>1$. Indeed, it is enough to verify that

$$
\forall^{*}\left(T_{0}, T_{1}, \ldots\right) \in[E]^{\mathbb{N}}\left(\left\{T_{n}\right\} \text { is dense in }[E]\right) .
$$

To see this, let $\left\{g_{n}\right\}$ be dense in $[E]$. Let $\delta_{u}(S, T)=\mu(\{x: S(x) \neq T(x)\})$ be the uniform metric on $\operatorname{Aut}(X, \mu)$. Then for any $\epsilon>0, n \in \mathbb{N}$,

$$
Y_{\epsilon, n}=\left\{\left(T_{0}, T_{1}, \ldots\right) \in[E]^{\mathbb{N}}: \exists i\left(d_{u}\left(T_{i}, g_{n}\right)<\epsilon\right)\right\}
$$

is open and dense in $[E]^{\mathbb{N}}$, thus

$$
Y=\bigcap_{\epsilon, n} Y_{\epsilon, n}
$$

is dense $G_{\delta}$ and clearly

$$
\left(T_{0}, T_{1}, \ldots,\right) \in Y \Rightarrow\left\{T_{n}\right\} \text { is dense in }[E] .
$$


Corollary 6.2 Let E be a countable, measure preserving, ergodic equivalence relation on $(X, \mu)$ with $C(E)>1$. Then for each $2 \leq n<\infty$, there is $\epsilon>0$ such that

$$
\left\{a \in \operatorname{AP}\left(\mathbb{F}_{n},[E]\right): C(a) \geq 1+\epsilon\right\}
$$

has non-empty interior in $\operatorname{AP}\left(\mathbb{F}_{n},[E]\right)$ with the uniform topology.

Proof. It is shown in Kechris [Ke09], Section 10, Remark following 10.14, that for infinite, finitely generated groups $\Gamma$ the function $a \mapsto C(a)$ on $A(\Gamma, X, \mu)$ is upper semicontinuous in the uniform topology. It follows that for each $\delta,\{a \in A(\Gamma, X, \mu): C(a) \geq \delta\}$ is uniformly closed. Thus

$$
\left\{a \in \operatorname{AP}\left(\mathbb{F}_{n},[E]\right): C(a)>1\right\}
$$

is non-meager in $\operatorname{AP}\left(\mathbb{F}_{n},[E]\right)$ and the union of the sequence of closed sets

$$
\left\{a \in \operatorname{AP}\left(\mathbb{F}_{n},[E]\right): C(a) \geq 1+\frac{1}{m}\right\}
$$

Thus for some $\epsilon=\frac{1}{m},\left\{a \in \operatorname{AP}\left(\mathbb{F}_{n},[E]\right): C(a) \geq 1+\epsilon\right\}$ has non-empty interior in $\operatorname{AP}\left(\mathbb{F}_{n},[E]\right)$.

This corollary shows that there are two elements $S, T \in[E], S$ aperiodic, such that $C\left(E_{S, T}\right) \geq 1+\epsilon$ and for any $S^{\prime}, T^{\prime} \in[E], S^{\prime}$ aperiodic, which are sufficiently close to $S, T$ in the uniform topology (i.e., differ by $S, T$ on a set of very small measure), we still have $C\left(E_{S^{\prime}, T^{\prime}}\right) \geq 1+\epsilon$.

In Kechris-Miller [KM], 28.8, it was shown, for $E$ as in 6.2 , that there is a free ergodic $a \in A\left(\mathbb{F}_{2},[E]\right)$. Then of course $C(a)=2$. It would be natural to think that an open nbhd of any such action would be a witness to the conclusion of the previous corollary. However, this is not the case in view of the following example due to Hjorth.

Proposition 6.3 (Hjorth) Let $\mathbb{F}_{n}=\left\langle\gamma_{1}, \gamma_{2}, \ldots\right\rangle$ be the free group with free generators $\gamma_{1}, \gamma_{2}, \ldots(1 \leq n \leq \infty)$. Let $a \in A\left(\mathbb{F}_{n}, X, \mu\right)$ be such that $\gamma_{1}^{a}$ is ergodic. Assume that there is an ergodic $U \in \operatorname{Aut}(X, \mu)$ which commutes with $a$, i.e., $U$ commutes with each $\gamma_{i}^{a}$. Then there is a sequence $a_{m} \in A\left(\mathbb{F}_{n}, X, \mu\right)$ with $\gamma_{1}^{a_{m}}$ ergodic, $C\left(a_{m}\right)=1$ and $a_{m} \rightarrow$ a uniformly.

Proof. We take $n=2$ for notational simplicity and let $\gamma_{1}^{a}=S, \gamma_{2}^{a}=T$.

First we note that $C\left(E_{S, T, U}\right)=1$; this follows from Kechris-Miller [KM], 24.8. It is thus enough to find $S_{m}, T_{m} \in \operatorname{Aut}(X, \mu)$ such that $S_{m}$ is ergodic, 
$S_{m} \rightarrow S, T_{m} \rightarrow T$ uniformly and $E_{S_{m}, T_{m}}=E_{S, T, U}$ (then $a_{m}$ given by $\gamma_{1}^{a_{m}}=$ $S_{m}, \gamma_{2}^{a_{m}}=T_{m}$ works).

Next we note the following fact: if $E$ is a countable, measure preserving equivalence relation, $E_{S} \subseteq E$ and $A=\{x: x E U(x)\}$ has positive measure, then $E_{U} \subseteq E$. Indeed, given any $x \in X$, by the ergodicity of $S$, there is $n \in \mathbb{Z}$ such that $S^{n}(x) \in A$ and so

$$
x E S^{n}(x) E U\left(S^{n}(x)\right)=S^{n}(U(x)) E U(x),
$$

so $E_{U} \subseteq E$. Similarly, if $E_{U} \subseteq E$ and $B=\{x: x E T(x)\}$ has positive measure, then $E_{T} \subseteq E$.

To find $S_{m}, T_{m}$, let first $A_{m} \subseteq X$ be a Borel set with $\mu\left(A_{m}\right)<1 / m$. Then $\mu\left(U\left(A_{m}\right)\right)=\mu\left(A_{m}\right)<1 / m$ and so if $C_{m}=\left\{x: T(x) \notin U\left(A_{m}\right)\right\}, \mu\left(C_{m}\right)>$ $1-1 / m$ and $T\left(C_{m}\right) \cap U\left(A_{m}\right)=\emptyset$. Let $B_{m}=C_{m} \cap\left(X \backslash A_{m}\right)$, so that $\mu\left(B_{m}\right)>1-2 / m$. Then, using the ergodicity of $U$, we can find $T_{m} \in\left[E_{U}\right]$ such that $T_{m}\left|B_{m}=T\right| B_{m}$ (thus $T_{m}, T$ differ only a set of measure $<2 / m$ ), and $T_{m}\left|A_{m}=U\right| A_{m}$. Let also $S_{m}=S$. Clearly $S_{m} \rightarrow S, T_{m} \rightarrow T$ uniformly. Let $E_{m}=E_{S_{m}, T_{m}}$. Then by the preceding fact, applied to $E_{m}$, we conclude that $E_{m} \supseteq E_{S, T, U}$ and since $T_{m} \in\left[E_{U}\right], E_{m}=E_{S_{m}, T_{m}} \subseteq E_{S, T, U}$, so $E_{m}=$ $E_{S, T, U}$.

Take now $n=2$ in 6.3 and a free, ergodic action $a \in A\left(\mathbb{F}_{2}, X, \mu\right)$ for which there is an ergodic $U \in \operatorname{Aut}(X, \mu)$, which commutes with $a$, and let $a_{m}$ be as in the conclusion of 6.3. Let $F$ be the equivalence relation generated by $a$ and $\left\{a_{m}\right\}$, and $E \supseteq F$ be an equivalence relation such that $C(E)>1$. Then no nbhd of $a$ is a witness to the conclusion of 6.2 for this $E$.

It is clear from 5.2 that $\left\{a \in A\left(\mathbb{F}_{n}, X, \mu\right): E_{a}\right.$ is aperiodic, hyperfinite $\}$ is weakly dense in $A\left(\mathbb{F}_{n}, X, \mu\right)$ and thus so if $\left\{a \in A\left(\mathbb{F}_{n}, X, \mu\right): C(a)=1\right\}(1 \leq$ $n \leq \infty)$. Recall that $E_{a}$ is aperiodic if (almost) all its equivalence classes are infinite. Using the preceding result we can actually prove a stronger statement.

Proposition 6.4 The set

$$
\left\{a \in A\left(\mathbb{F}_{n}, X, \mu\right): E_{a} \text { is not hyperfinite \& } C(a)=1\right\}
$$

is weakly dense in $A\left(\mathbb{F}_{n}, X, \mu\right)(2 \leq n \leq \infty)$.

Proof. Again we take $n=2$ for notational simplicity.

Fix $a_{0} \in A\left(\mathbb{F}_{2}, X, \mu\right)$ and a weak nbhd $U$ of $a$ in order to find $a \in U$ with $E_{a}$ not hyperfinite and $C(a)=1$. 
By Kechris [Ke09], Section 10, (G), we can assume that there is an action $b_{0} \in A\left(\mathbb{F}_{2} \times \mathbb{Z}, X, \mu\right)$ with $a_{0}=b_{0} \mid \mathbb{F}_{2}$. Now $\mathbb{F}_{2} \times \mathbb{Z}$ has the Haagerup Approximation Property (HAP), so by Hjorth [Hj08] there is a mixing action $b_{0}^{\prime} \in A\left(\mathbb{F}_{2} \times \mathbb{Z}, X, \mu\right)$ as close as we want to $b_{0}$ in the weak topology. Let $c_{0}$ be a free, mixing action in $A\left(\mathbb{F}_{2} \times \mathbb{Z}, X, \mu\right)$ and consider $b_{0}^{\prime} \times c_{0}$. It is free, mixing and $b_{0}^{\prime} \prec b_{0}^{\prime} \times c_{0}$, i.e., there is an isomorphic copy of $b_{0}^{\prime} \times c_{0}$ as close as we want to $b_{0}^{\prime}$ in the weak topology. Thus there is a free mixing action $d_{0} \in A\left(\mathbb{F}_{2} \times \mathbb{Z}, X, \mu\right)$ such that $d_{0} \mid \mathbb{F}_{2} \in U$. Then, by $6.3, d_{0} \mid \mathbb{F}_{2}$ is the uniform limit of a sequence of actions $a_{m} \in A\left(\mathbb{F}_{2}, X, \mu\right)$ with $C\left(a_{m}\right)=1$.

Lemma 6.5 For any infinite, countable group $\Gamma$, the set $\left\{a \in A(\Gamma, X, \mu): E_{a}\right.$ is hyperfinite\} is uniformly closed.

Granting this, since clearly $E_{d_{0} \mid \mathbb{F}_{2}}$ is not hyperfinite, it follows that we can also assume that $E_{a_{m}}$ is not hyperfinite and the proof is complete.

Proof of 6.5. Let $a_{n} \in A(\Gamma, X, \mu), E_{a_{n}}$ hyperfinite and $a_{n} \rightarrow a \in$ $A(\Gamma, X, \mu)$ uniformly. Let $E_{n}=E_{a_{n}}, E=E_{a}$. Clearly for each $n, F_{n}=$ $\bigcap_{m>n} E_{m}$ is hyperfinite and $F_{0} \subseteq F_{1} \subseteq \ldots$, so

$$
\bigcup_{n} F_{n}=\bigcup_{n} \bigcap_{m>n} E_{m}
$$

is hyperfinite. It is thus enough to check that $E \subseteq \bigcup_{n} \bigcap_{m>n} E_{n}$. If not, there is $\gamma \in \Gamma$ and a set of positive measure $A$ such that

$$
x \in A \Rightarrow\left(x, \gamma^{a}(x)\right) \notin \bigcup_{n} \bigcap_{m>n} E_{m} .
$$

i.e., for $x \in A$ and for infinitely many $n,\left(x, \gamma^{a}(x)\right) \notin E_{n}$. Now $\gamma^{a_{n}} \rightarrow \gamma^{a}$ uniformly, so for all large enough $n, \mu\left(\left\{x \in A: \gamma^{a}(x)=\gamma^{a_{n}}(x)\right\}\right)>0$, a contradiction.

(B) There have been some very interesting recent results that have the following general form: Let $P$ be a property of countable, measure preserving equivalence relations on $(X, \mu)$, (which we intuitively think as strongly violating hyperfiniteness). Then there exists an ergodic equivalence relation $E$ such that every ergodic $F \subseteq E$ either is hyperfinite or else has property P. Chifan-Ioana [CI] show that for $P$ being the property of strong ergodicity (also called $E_{0}$-ergodicity), this holds for the equivalence relation $E$ induced by the shift action of $\Gamma$ on $[0,1]^{\Gamma}$ for any infinite, countable group $\Gamma$. Ozawa 
[O] showed that the same holds when $E$ is the equivalence relation induced by the action of $\mathrm{SL}_{2}(\mathbb{Z})$ on $\mathbb{T}^{2}$. Finally, Ioana [I09] showed that for $P$ being the property of being rigid, this holds again for the equivalence relation induced by the action of $\mathrm{SL}_{2}(\mathbb{Z})$ on $\mathbb{T}^{2}$.

Let us note here that for pairs $P, E$ satisfying the above dichotomy,we have the following density result.

Proposition 6.6 Let $P$ be a property of countable, measure preserving equivalence relations that implies non-hyperfiniteness. Let $E$ be a countable, measure preserving, ergodic equivalence relation which is not hyperfinite such that every ergodic subequivalence relation $F \subseteq E$ is either hyperfinite or has property $P$. Then $\left\{a \in A\left(\mathbb{F}_{n},[E]\right): E_{a}\right.$ has property $\left.P\right\}$ is uniformly dense in $\operatorname{AP}\left(\mathbb{F}_{n},[E]\right), 2 \leq n \leq \infty$.

Proof. Take again $n=2$ for notational simplicity. As in the proof of 6.1 , let $S_{0} \in[E]$ be ergodic such that $E_{S_{0}}$ is maximal under inclusion. Fix $(S, T) \in \operatorname{AP}\left(\mathbb{F}_{2},[E]\right)$ and uniform nbhds $U, V$ of $S, T$, resp. Since we can find $K \in[E]$ such that $K S_{0} K^{-1} \in U$, we may as well assume that $S_{0} \in U$. Now $[E] \backslash\left[E_{S_{0}}\right]$ is uniformly dense in $[E]$, so let $T_{0} \in[E] \backslash\left[E_{S_{0}}\right]$ be in $V$. Then $E_{S_{0}, T_{0}}$ is ergodic and non-hyperfinite, by the maximality of $E_{S_{0}}$, and thus has property $P$, which completes the proof.

Corollary 6.7 In the context of 6.6, $\left\{a \in A\left(\mathbb{F}_{n}, X, \mu\right): E_{a}\right.$ has property $\left.P\right\}$ is weakly dense in $A\left(\mathbb{F}_{n}, X, \mu\right)$.

It follows, for example, that the strongly ergodic (resp., rigid) actions are weakly dense in $A\left(\mathbb{F}_{n}, X, \mu\right), 2 \leq n \leq \infty$, a fact that can be also proved more directly as pointed out in Ioana [I09], 6.2. Also Abert informed me that he has shown, by a different method, that the actions in $A\left(\mathbb{F}_{n}, X, \mu\right)$ that have spectral gap are weakly dense.

\section{A Appendix. Some facts about co-induced actions}

Suppose that a countable group $\Delta$ acts on a countable set $T$ and let $\sigma: \Delta \times$ $T \rightarrow \operatorname{Aut}(X, \mu)$ be a cocycle of the action of $\Delta$ on $T$ with values in $\operatorname{Aut}(X, \mu)$, i.e., a map satisfying the property

$$
\sigma\left(\delta_{1} \delta_{2}, t\right)=\sigma\left(\delta_{1}, \delta_{2} \cdot t\right) \sigma\left(\delta_{2}, t\right)
$$


for $\delta_{1}, \delta_{2} \in \Delta, t \in T$. We define an action of $\Delta$ on $(Y, \nu)$, where $Y=X^{T}, \nu=$ $\mu^{T}$ (the product measure), by

$$
(\delta \cdot f)(t)=\sigma\left(\delta^{-1}, t\right)^{-1}\left(f\left(\delta^{-1} \cdot t\right)\right) .
$$

It is easily checked that this is a measure preserving action of $\Delta$ on $(Y, \nu)$.

If $\tau: \Delta \times T \rightarrow \operatorname{Aut}(X, \mu)$ is another cocycle which is cohomologous to $\sigma$, i.e., there is $f: T \rightarrow \operatorname{Aut}(X, \mu)$ such that $\tau(\delta, t)=f(\delta \cdot t) \sigma(\delta, t) f(t)^{-1}$, then the action induced by $\tau$ is isomorphic to the action induced by $\sigma$ via the map $\varphi: Y \rightarrow Y$ given by

$$
\varphi(p)(t)=f(t)(p(t))
$$

Let now $\Gamma \leq \Delta$ be a subgroup and let $T$ be a transversal for the left cosets of $\Gamma$ with $1 \in T$. Let $\Delta$ act on $T$ by defining $\delta \cdot t$ to be the unique element of $T$ in $\delta t \Gamma$ and let $\rho: \Delta \times T \rightarrow \Gamma$ be the cocycle defined by

$$
(\delta \cdot t) \rho(\delta, t)=\delta t
$$

If $a \in A(\Gamma, X, \mu)$, then $\rho$ gives rise to the cocycle $\sigma: \Delta \times T \rightarrow \operatorname{Aut}(X, \mu)$ defined by $\sigma(\delta, t)=\rho(\delta, t)^{a}$. We call the action on $(Y, \nu)$ given by $\sigma$ the co-induced action of $a$, in symbols

$$
\operatorname{CInd}_{\Gamma}^{\Delta}(a)
$$

Thus $b=\operatorname{CInd}_{\Gamma}^{\Delta}(a)$ is the action of $\Delta$ on $\left(X^{T}, \mu^{T}\right)$ given by

$$
(\delta \cdot f)(t)=\rho\left(\delta^{-1}, t\right)^{-1} \cdot f\left(\delta^{-1} \cdot t\right)
$$

where the action on the right-hand side is the action $a$.

Note that $T$ can be identified with $\Delta / \Gamma$ and thus $\left(X^{T}, \mu^{T}\right)$ with the space $\left(X^{\Delta / \Gamma}, \mu^{\Delta / \Gamma}\right)$. The action of $\Delta$ on $T$ becomes then the usual action $\delta \cdot \delta^{\prime} \Gamma=\delta \delta^{\prime} \Gamma$ of $\Delta$ on $\Delta / \Gamma$.

Various properties of the co-induced action are given in Ioana [I07] and Kechris [Ke09], Section 10, (G). We record a few that we use in this paper:

(i) $a \sqsubseteq \operatorname{CInd}_{\Gamma}^{\Delta}(a) \mid \Gamma$, in fact the map $f \mapsto f(1)$ demonstrates that $a$ is a factor of $\operatorname{CInd}_{\Gamma}^{\Delta}(a) \mid \Gamma$.

$$
\begin{gathered}
a \mapsto \operatorname{CInd}_{\Gamma}^{\Delta}(a) \\
A(\Gamma, X, \mu) \rightarrow A(\Delta, Y \nu)
\end{gathered}
$$

is continuous in the weak topology. 
(iii) $a \cong b \Rightarrow \operatorname{CInd}_{\Gamma}^{\Delta}(a) \cong \operatorname{CInd}_{\Gamma}^{\Delta}(b)$.

We prove below some further properties of the co-induced action that we also need in this paper.

Proposition A.1 Co-inducing preserves weak containment, i.e.,

$$
a \prec b \Rightarrow \operatorname{CInd}_{\Gamma}^{\Delta}(a) \prec \operatorname{CInd}_{\Gamma}^{\Delta}(b) .
$$

Proof. Since $a \prec b$, there is a sequence $b_{n}$ such that $b_{n} \cong b$ and $b_{n} \rightarrow$ $a$ weakly. Then $\operatorname{CInd}_{\Gamma}^{\Delta}\left(b_{n}\right) \cong \operatorname{CInd}_{\Gamma}^{\Delta}(b)$ and by (ii) above $\operatorname{CInd}_{\Gamma}^{\Delta}\left(b_{n}\right) \rightarrow$ $\operatorname{CInd}_{\Gamma}^{\Delta}(a), \operatorname{so} \operatorname{CInd}_{\Gamma}^{\Delta}(a) \prec \operatorname{CInd}_{\Gamma}^{\Delta}(b)$.

If $\Gamma \leq \Delta$ and $(X, \mu)$ is a measure space (perhaps with atoms), we denote by $s_{\Delta, \Delta / \Gamma, X}$ the shift action of $\Delta$ on $X^{\Delta / \Gamma}$ corresponding to the canonical action of $\Delta$ on $\Delta / \Gamma$ :

$$
\left(\delta_{1} \cdot f\right)\left(\delta_{2} \Gamma\right)=f\left(\delta_{1}^{-1} \delta_{2} \Gamma\right) .
$$

If $X=2=\{0,1\}$ and $\mu(\{0\})=\mu(\{1\})=1 / 2$, we simply write $s_{\Delta, \Delta / \Gamma}$. If $T$ is a transversal for $\Delta / \Gamma$ with $1 \in T$, then $\Delta / \Gamma$ can be identified with $T$ and $s_{\Delta, \Delta / \Gamma, X}$ is the action

$$
(\delta \cdot f)(t)=f\left(\delta^{-1} \cdot t\right)
$$

where $\Delta$ acts on $T$ in the previous sense.

Proposition A.2 Let $H \leq \Gamma \leq \Delta$ and $(X, \mu)$ a measure space (perhaps with atoms). Then

$$
\operatorname{CInd}_{\Gamma}^{\Delta}\left(s_{\Gamma, \Gamma / H, X}\right) \cong s_{\Delta, \Delta / H, X}
$$

Proof. Fix a transversal $S$ for the left cosets of $H$ in $\Gamma$ containing 1 and a transversal $T$ for the left cosets of $\Gamma$ in $\Delta$ containing 1 . Then $T S=\{t s: t \in$ $T, s \in S\}$ is a transversal for the left cosets of $H$ in $\Delta$.

The action $s_{\Gamma, \Gamma / H, X}$ is the action of $\Gamma$ on $X^{\Gamma / H}=X^{S}$ defined by

$$
\gamma \cdot p(s)=p\left(\gamma^{-1} \cdot s\right)
$$

where $\Gamma$ acts on $S$ by $\gamma \cdot s=$ (the unique element of $S$ in the coset $\gamma s H$ ). Let also $\sigma: \Gamma \times S \rightarrow H$ be the associated cocycle given by $(\gamma \cdot s) \sigma(\gamma, s)=\gamma s$.

The action $\operatorname{CInd}_{\Gamma}^{\Delta}\left(s_{\Gamma, \Gamma / H, X}\right)$ is the action of $\Delta$ on $\left(X^{\Gamma / H}\right)^{\Delta / \Gamma}=\left(X^{S}\right)^{T}$, given by

$$
\delta \cdot q(t)=\rho\left(\delta^{-1}, t\right)^{-1} \cdot q\left(\delta^{-1} \cdot t\right)
$$


where $\Delta$ acts on $T$ in the usual way and $\rho: \Delta \times T \rightarrow \Gamma$ is the associated cocycle given by $(\delta \cdot t) \rho(\delta, t)=\delta t$.

The measure spaces $\left(\left(X^{S}\right)^{T},\left(\mu^{S}\right)^{T}\right)$ and $\left(X^{S \times T}, \mu^{S \times T}\right)$ are isomorphic via the map $p \mapsto \varphi(p)=q$, where $q(s, t)=p(t)(s)$. Clearly $(s, t) \mapsto t s$ is a bijection of $S \times T$ with $T S$ and thus $X^{S \times T}$ is identified with $X^{T S}=$ $X^{\Delta / H}$. Therefore $\psi:\left(X^{S}\right)^{T} \rightarrow X^{T S}$, given by $\psi(p)=q$, where $q(t s)=$ $\varphi(p)(s, t)=p(t)(s)$, is an isomorphism of $\left(\left(X^{S}\right)^{T},\left(\mu^{S}\right)^{T}\right)$ with $\left(X^{T S}, \mu^{T S}\right)=$ $\left(X^{\Delta / H}, \mu^{\Delta / H}\right)$ and we will show that it sends $\operatorname{CInd}_{\Gamma}^{\Delta}\left(s_{\Gamma, \Gamma / H, X}\right)$ to $s_{\Delta, \Delta / H, X}$.

We have $(\delta \cdot p)(t)=\rho\left(\delta^{-1} \cdot t\right)^{-1} \cdot p\left(\delta^{-1}, t\right)$, so

$$
\begin{aligned}
\psi(\delta \cdot p)(t s)=(\delta \cdot p)(t)(s) & =\rho\left(\delta^{-1} \cdot t\right)^{-1} \cdot p\left(\delta^{-1} \cdot t\right)(s) \\
& =p\left(\delta^{-1} \cdot t\right)\left(\rho\left(\delta^{-1} \cdot t\right) \cdot s\right) \\
& =\psi(p)\left(\left(\delta^{-1} \cdot t\right)\left(\rho\left(\delta^{-1}, t\right) \cdot s\right)\right) .
\end{aligned}
$$

On the other hand,

$$
(\delta \cdot \psi(p))(t s)=\psi(p)\left(\delta^{-1} \cdot t s\right)
$$

Now $\left(\delta^{-1} \cdot t\right) \rho\left(\delta^{-1}, t\right)=\delta^{-1} t$, so $\delta^{-1} t s=\left(\delta^{-1} \cdot t\right) \rho\left(\delta^{-1}, t\right) s$, thus if we put $\gamma=\rho\left(\delta^{-1}, t\right) \in \Gamma$, we have

$$
\begin{aligned}
\delta^{-1} t s & =\left(\delta^{-1} \cdot t\right) \gamma s \\
& =\left(\delta^{-1} \cdot t\right)(\gamma \cdot s) \sigma(\gamma, s) \\
& =\left(\delta^{-1} \cdot t\right)\left(\rho\left(\delta^{-1}, t\right) \cdot s\right) \sigma(\gamma, s)
\end{aligned}
$$

and $\sigma(\gamma, s) \in H$, thus

$$
\delta^{-1} \cdot t s=\left(\delta^{-1} \cdot t\right)\left(\rho\left(\delta^{-1}, t\right) \cdot s\right)
$$

so

$$
\delta \cdot \psi(p)(t s)=\psi(p)\left(\left(\delta^{-1} \cdot t\right)\left(\rho\left(\delta^{-1}, t\right) \cdot s\right)\right),
$$

i.e., $\psi(\delta \cdot p)=\delta \cdot \psi(p)$, and the proof is complete.

Assume $\Gamma \leq \Delta$ and let now $a \in A(\Delta, X, \mu)$ be an action of the bigger group $\Delta$. One can form the restriction $a \mid \Gamma \in A(\Gamma, X, \mu)$ and then co-induce that to get $\operatorname{CInd}_{\Gamma}^{\Delta}(a \mid \Gamma)$. We will describe below this action.

Consider first the (diagonal) product action $a^{\Delta / \Gamma}$ on $\left(X^{\Delta / \Gamma}, \mu^{\Delta / \Gamma}\right)=$ $\left(X^{T}, \mu^{T}\right)$ :

$$
\delta \cdot f=(t \mapsto \delta \cdot f(t)),
$$


where the action on the right-hand side is the action $a$. We also have the shift action $s_{\Delta, \Delta / \Gamma, X}$ on $\left(X^{\Delta / \Gamma}, \mu^{\Delta / \Gamma}\right)$. Note that these actions commute, i.e., for each $\delta_{1}, \delta \in \Delta$

$$
\delta_{1}^{a^{\Delta / \Gamma}} \delta_{2}^{s_{\Delta, \Delta / \Gamma, X}}=\delta_{2}^{s_{\Delta, \Delta / \Gamma, X}} \delta_{1}^{a^{\Delta / \Gamma}},
$$

so we can define a new action, denoted by $a^{\Delta / \Gamma} \circledast s_{\Delta, \Delta / \Gamma}$, which is given by

$$
\delta \cdot f(t)=\delta \cdot f\left(\delta^{-1} \cdot t\right)
$$

Proposition A.3 For $\Gamma \leq \Delta$ and for each action $a \in A(\Delta, X, \mu)$,

$$
\operatorname{CInd}_{\Gamma}^{\Delta}(a) \cong a^{\Delta / \Gamma} \circledast s^{\Delta, \Delta / \Gamma, X} .
$$

Proof. Going back to the beginning of this Appendix, let the cocycle $\sigma_{1}: \Delta \times T \rightarrow \operatorname{Aut}(X, \mu)$ be given by $\sigma_{1}(\delta, t)=\delta^{a}$. Let also $\sigma_{2}(\delta, t)=\rho(\delta, t)^{a}$. Then the action of $\Delta$ on $\left(X^{T}, \mu^{T}\right)$ corresponding to $\sigma_{1}$ is $a^{\Delta / \Gamma} \circledast s^{\Delta, \Delta / \Gamma, X}$, while the action corresponding to $\sigma_{2}$ is $\operatorname{CInd}_{\Gamma}^{\Delta}(a)$. It is thus enough to show that $\sigma_{1}, \sigma_{2}$ are cohomologous, i.e., there is $f: T \rightarrow \operatorname{Aut}(X, \mu)$ such that $\sigma_{2}(\delta, t)=f(\delta \cdot t) \sigma_{1}(\delta, t) f(t)^{-1}$. By definition we have

$$
\sigma_{2}(\delta, t)=\rho(\delta, t)^{a}
$$

where $\rho(\delta, t)=(\delta \cdot t)^{-1} \delta t$, thus

$$
\begin{aligned}
\sigma_{2}(\delta, t) & =\left((\delta \cdot t)^{-1}\right)^{a} \delta^{a} t^{a} \\
& =\left((\delta \cdot t)^{-1}\right)^{a} \sigma_{1}(\delta, t) t^{a},
\end{aligned}
$$

SO

$$
f(t)=\left(t^{-1}\right)^{a}
$$

works.

If $\Gamma \leq \Delta$, then the action of $\Delta$ on $\Delta / \Gamma$ is amenable if it admits a finitely additive invariant probability measure (defined on all subsets of $\Delta / \Gamma$ ). For more about these actions, see, e.g., Glasner-Monod [GM] and KechrisTsankov $[\mathrm{KT}]$. We conclude this appendix with the following question.

Problem A.4 Let $\Gamma \leq \Delta$ and assume that the action of $\Delta$ on $\Delta / \Gamma$ is amenable. Is it true that for any $a \in A(\Delta, X, \mu)$,

$$
a \prec \operatorname{CInd}_{\Gamma}^{\Delta}(a \mid \Gamma) ?
$$


Note the assumption that the action of $\Delta$ on $\Delta / \Gamma$ is amenable is necessary because if $a=i_{\Delta}$ is the trivial action of $\Delta$ on $(X, \mu)$, then $\operatorname{CInd}_{\Gamma}^{\Delta}(a \mid \Gamma) \cong$ $s_{\Delta, \Delta / \Gamma, X}$, and $i_{\Delta} \prec s_{\Delta, \Delta / \Gamma, X}$ implies the amenability of the action of $\Delta$ on $\Delta / \Gamma$ (see Kechris-Tsankov $[\mathrm{KT}]$ ).

By extending the arguments in Kechris-Tsankov [KT], we can show that A.4 has a positive answer in certain cases, e.g., when $a=i_{\Delta}$ or $a=s_{\Delta, \Delta / H, X}$ (for any $H \leq \Delta$ ), but the general case remains opens.

\section{References}

[AE] M. Abert and G. Elek, Dynamical properties of profinite actions, preprint, 2008.

[AN] M. Abert and N. Nikolov, Rank gradient, cost of groups and the rank versus Heegard genus problem, arXiv:math/0701925, 2008.

[AW] M. Abert and B. Weiss, forthcoming.

[BdlH] M.B. Bekka and P. de la Harpe, Irreducibility of unitary group representations and reproducing kernel Hilbert spaces, Expo. Math., 21 (2003), 115-149.

[CI] I. Chifan and A. Ioana, Ergodic subequivalence relations induced by a Bernoulli action, arXiv:0802.2998, 2008.

[FM] J. Feldman and C.C. Moore, Ergodic equivalence relations, cohomology and von Neumann algebras, I, Trans. Amer. Math. Soc., 234(2) (1977), 289-324.

[G] D. Gaboriau, Coût des relations d'equivalence et des groupes, Inv. Math., 139 (2000), 41-98.

[GM] Y. Glasner and N. Monod, Amenable actions, free products and a fixed point property, Bulletin London Math. Soc., 39(1) (2007), $138-150$.

[GTW] E. Glasner, J.-P. Thouvenot and B. Weiss, Every countable group has the weak Rokhlin property, Bull. London Math. Soc., 38(6) (2006), 932-936. 
[GN] R.I. Grigorchuk and V.V. Nekrashevych, Amenable actions of nonamenable groups, J. Math. Sci. (N.Y.), 140(3) (2007), 391-397.

[Hj02] G. Hjorth, A converse to Dye's Theorem, Trans. Amer. Math. Soc. 357(8) (2002), 3083-3103.

[Hj08] G. Hjorth, Groups with HAP, preprint, 2008. (posted in http://math.ucla.edu/ greg/HAP.pdf)

[I07] A. Ioana, Orbit inequivalent actions for groups containing a copy of $\mathbb{F}_{2}$, arXiv:math/0701027, 2007.

[I09] A. Ioana, Relative property (T) for the subequivalence relations induced by the actions of $\mathrm{SL}_{2}(\mathbb{Z})$ on $\mathbb{T}^{2}$, arXiv:0901.1874, 2009.

[IPP] A. Ioana, J. Peterson and S. Popa, Amalgamated free products of $w$ rigid factors and calculation of their symmetry groups, Acta Math., 200(1) (2008), 85-153.

[Ke07] A.S. Kechris, Unitary representations and modular actions, J. Math. Sc., 140(3) (2007), 398-425.

[Ke09] A.S. Kechris, Global aspects of ergodic group actions, preprint, 2009. (posted in http://www.math.caltech.edu/people/kechris.html)

[KM] A.S. Kechris and B. Miller, Topics in Orbit Equivalence, Lecture Notes in Math. 1852, 2004.

[KT] A.S. Kechris and T.Tsankov, Amenable actions and almost invariant sets, Proc. Amer. Math. Soc., 136(2) (2007), 687-697.

[LSh] A. Lubotzky and Y. Shalom, Finite representations in the unitary dual and Ramanujan groups, Contemp. Math., 347 (2004), 173-189.

[LZ] A. Lubotzky and A. Zuk, On property $(\tau)$, preprint, 2003. (posted in http://www.ma.huji.ac.il/ alexlub/, 2003)

[LS] R.C. Lyndon and P.E. Schupp, Combinatorial Group Theory, Springer, 2001.

[O] N. Ozawa, An example of a solid von Neumann algebra, arXiv:0706.3623, 2007. 
[vD] E.K. van Douwen, Measures invariant under actions of $F_{2}$, Topology Appl., 34(1) (1990), 53-68.

[Y] H. Yoshizawa, Some remarks on unitary representations of the free group, Osaka Math. J., 3 (1951), 55-63.

[Z] R. Zimmer, Ergodic Theory and Semisimple Groups, Birkhäuser, 1984.

Department of Mathematics

California Institute of Technology

Pasadena, CA 91125

kechris@caltech.edu 